\title{
5-O-Mycaminosyltylonolide antibacterial derivatives: design, synthesis and bioactivity
}

\author{
Akihiro Sugawara $^{1}$, Hitomi Maruyama ${ }^{1}$, Sho Shibusawa ${ }^{1}$, Hidehito Matsui ${ }^{1}$, Tomoyasu Hirose ${ }^{1}$, \\ Ayumi Tsutsui ${ }^{1}$, Robrecht Froyman ${ }^{2}$, Carolin Ludwig ${ }^{2}$, Johannes Koebberling ${ }^{2}$, Hideaki Hanaki ${ }^{1}$, \\ Gerd Kleefeld $^{2}$, Satoshi Ōmura ${ }^{1}$ and Toshiaki Sunazuka ${ }^{1}$
}

Tylosin is a 16-membered macrolide broad-spectrum antibiotic that has an important role in veterinary medicine, active against Gram-positive and a restricted range of Gram-negative bacteria. We synthesized 15 types of tylosin-related derivatives by chemical modification and evaluated them against mastitis pathogens. Among them, 20-deoxy-20-\{N-methyl- $N$-[1-(3-quinolyl)$1 H$-1,2,3-triazol-4-yl]methylamino\}-5-O-mycaminosyltylonolide 2 f and 20-deoxy-20-\{ $N$-benzyl- $N$-[1-(3-quinolyl)-1 $H$-1,2,3-triazol4-yl]methylamino\}-5-O-mycaminosyltylonolide $2 \mathrm{k}$ were found to not only expand their antibacterial impact to include Gramnegative bacteria, such as Escherichia coli and Klebsiella pneumoniae, but also to retain or increase antibacterial activity against Gram-positive bacteria, such as Staphylococcus aureus and Streptococcus uberis in comparison with the parent tylosin. The Journal of Antibiotics (2017) 70, 878-887; doi:10.1038/ja.2017.61; published online 31 May 2017

\section{INTRODUCTION}

Macrolide antibiotics are recognized as being essential medicines in both human and animal health worldwide. ${ }^{1}$ In particular, the 16-membered macrolide, tylosin (TYL, TYLAN ${ }^{\circledR}$, Figure 1), which was developed by Eli Lilly (Indianapolis, IN, USA), has a significant role in the treatment of infectious diseases, for example, respiratory diseases, mastitis, etc. in animal health. ${ }^{2}$ Our research group has been striving to create such antibiotics through chemical modification of naturally occurring microbial metabolites. ${ }^{3}$ Thirty years ago, our collaborative research with Eli Lilly resulted in the development of a novel antibiotic, tilmicosin (TLM, MICOTIL $\left.{ }^{\circledR}\right),{ }^{4}$ (http://www.elanco.us/products-services/beef/cattle-brd.aspx) a tylosin derivative, for respiratory diseases of animals. More recently, the Institute of Microbial Chemistry and Merck Animal Health developed a novel antibiotic tildipirosin (ZUPREVO $\left.{ }^{\circledR}\right)^{5}$ (http://www.zuprevo.com) for prevention of bovine respiratory disease, which possesses potent antibacterial activity against Mannheimia haemolytica, Pasteurella multocida and Histophilus somni. However, the antibacterial activity of these compounds against Escherichia coli and Klebsiella pneumoniae, which occasionally cause mastitis, are still unsatisfactory. Mastitis is an infectious disease, found in domestic animals and humans, caused by bacteria, such as Staphylococcus aureus, Streptococcus uberis, E. coli and $K$. pneumoniae. In the dairy industry it is a major problem, leading to lost milk production and substantial economic losses for farmers. ${ }^{6}$ Although some antibiotics such as cephem ${ }^{7}$ and lincosamide ${ }^{8}$ have been used, novel antibiotics are still urgently needed. Therefore, our continuing research efforts have been focused on creating antibiotic macrolides with an expanded spectrum of activity, in particular against Gram-negative bacteria such as E. coli.
In this study, we report that 16-membered macrolide tylosin derivatives, 20-deoxy-20-\{ $N$-methyl- $N$-[1-(3-quinolyl)-1H-1,2,3-triazol4-yl]methylamino -5 -O-mycaminosyltylonolide $2 \mathrm{f}$ and 20-deoxy-20$\{\mathrm{N}$-benzyl- $\mathrm{N}$-[1-(3-quinolyl)-1H-1,2,3-triazol-4-yl]methylamino\}-5-Omycaminosyltylonolide $\mathbf{2 k}$, were found to not only exhibit significant activity against E. coli and $K$. pneumoniae but also maintained or increased their antibacterial activity against Gram-positive bacteria.

\section{RESULTS AND DISCUSSION}

Our primary approach was to introduce a functionalized amino moiety, based on 5-O-mycaminosyltylonolide (OMT), ${ }^{9}$ in order to increase antibacterial activity, as our structure-activity relationships analyses of 16-membered macrolides were as follows (Table 1, we re-evaluated antibacterial activity of these natural products and their derivatives using our assay system): (1) OMT (MIC $128 \mu \mathrm{g} \mathrm{ml}^{-1}$ against E. coli, $64 \mu \mathrm{g} \mathrm{ml}^{-1}$ against $K$. pneumoniae) shows slightly better antibacterial activity against $E$. coli and $K$. pneumoniae than that of tylosin (MIC $>128 \mu \mathrm{g} \mathrm{ml}^{-1}$ against E. coli, $>128 \mu \mathrm{g} \mathrm{ml}^{-1}$ against K. pneumoniae), along with similar antibacterial activity against Grampositive bacteria, for example, S. aureus; (2) introduction of amino groups (tildipirosin; MIC $8 \mu \mathrm{g} \mathrm{ml}^{-1}$ against E. coli, $16 \mu \mathrm{g} \mathrm{ml}^{-1}$ against $K$. pneumoniae) shows increased antibacterial activity against $E$. coli and $K$. pneumoniae compared with that of OMT (MIC $128 \mu \mathrm{g} \mathrm{ml}^{-1}$ against E. coli, $64 \mu \mathrm{g} \mathrm{ml}^{-1}$ against K. pneumoniae).

However, tildipirosin (MIC 8-16 $\mu \mathrm{g} \mathrm{m}^{-1}$ against S. aureus) does not show stronger activity against Gram-positive bacteria than OMT (MIC $1 \mu \mathrm{g} \mathrm{ml}^{-1}$ against $S$. aureus). This means that introduction of an amino group to OMT loses antibacterial activity against Gram-positive bacteria. With initial structure-activity relationshi in mind, we decided

${ }^{1}$ Kitasato Institute for Life Sciences and Graduate School of Infection Control Sciences, Kitasato University, Tokyo, Japan and ${ }^{2}$ Bayer Animal Health GmbH, Leverkusen, Germany Correspondence: Professor Satoshi Ōmura or Professor Toshiaki Sunazuka, Kitasato Institute for Life Sciences and Graduate School of Infection Control Sciences, Kitasato University, 5-9-1, Shirokane, Minato-ku, Tokyo 108-8641, Japan.

E-mail: omuras@insti.kitasato-u.ac.jp or sunazukatoshiaki@yahoo.co.jp

Received 20 December 2016; revised 9 March 2017; accepted 5 April 2017; published online 31 May 2017 



$\mathbf{R}_{1}$

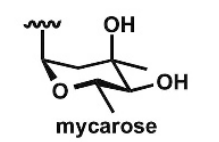

H

H

$\mathbf{R}_{2}$

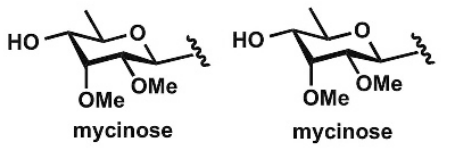

$\mathbf{H}$
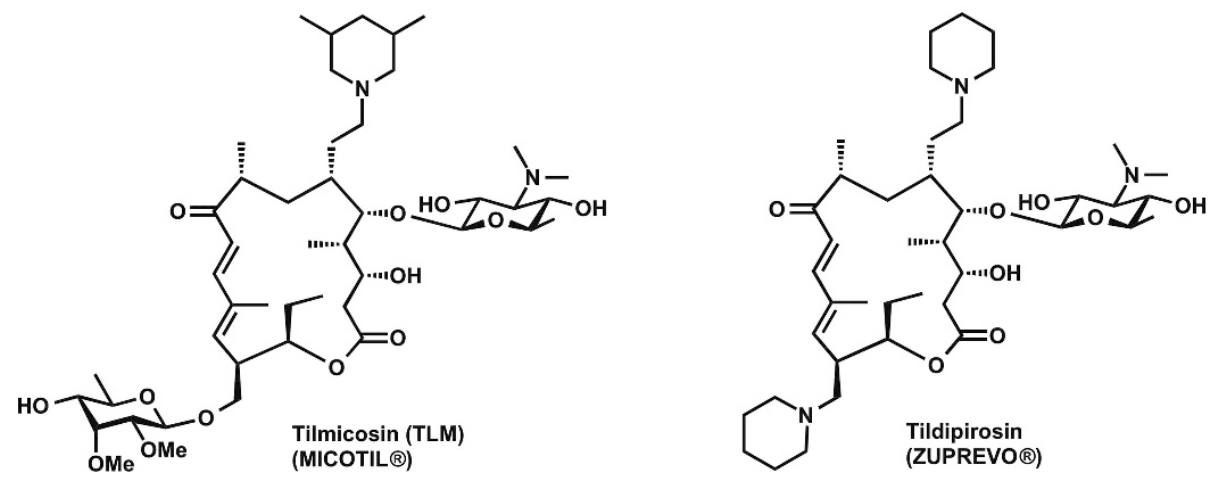

Figure 1 Structures of TYL and related analogues, Desmycosin, OMT, Tilmicosin and Tildipirosin.

to introduce not only some amino groups but also some hydrophobic groups ${ }^{10}$ into macrolides.

\section{Synthesis and biological evaluation}

At the outset, our efforts focused on making derivatives that introduced functionalized amino groups at the C20 position of OMT. We also envisioned that the copper-catalyzed triazole formation $^{11,12}$ of an $\mathrm{N}$-propargyl compound would allow us to readily search for an appropriate functional group, despite the fact that tylosin has many reactive functional groups, such as alcohol, enone, ester and dimethylamino groups. ${ }^{13,14}$ Consequently, we began to investigate appropriate functional groups via a triazole linker, utilizing coppercatalyzed triazole formation, in order to increase antibacterial activity against Gram-positive and -negative bacteria. Reductive amination $\left(\mathrm{NaBH}(\mathrm{OAc})_{3}, \mathrm{AcOH}, \mathrm{ClCH}_{2} \mathrm{CH}_{2} \mathrm{Cl}\right)$ of $\mathrm{OMT}$, synthesized under acidic condition ( $0.5 \mathrm{M}$ trifluoroacetic acid (TFA) in $\mathrm{H}_{2} \mathrm{O}$, reflux) from tylosin, ${ }^{9}$ with $\mathrm{N}$-methylpropargylamine (commercially available), afforded 1a in 93\% yield (Scheme 1). Copper-catalyzed triazole formation (tetrakis(acetonitrile) copper(I) hexafluorophosphate, tris [(1-benzyl-1H-1,2,3-triazol-4-yl)methyl]amine, $\mathrm{MeOH}$, room temperature) of 1a with benzyl azide (commercially available), adamantylazide (commercially available), phenyl azide (commercially available), 3 -azidopyridine, ${ }^{15}$ 2-azidonaphthalene ${ }^{16}$ and 3 -azidoquinoline ${ }^{17}$ readily led to the corresponding triazole products $2 \mathbf{a}$ (71\% yield), $\mathbf{2 b}(87 \%$ yield), 2 c ( $100 \%$ yield), 2 d (100\% yield), 2 e (76\% yield) and 2 f $(96 \%$ yield), respectively (Scheme 1). Although we will discuss about biological evaluation later, Table 2 shows that 2 f displayed better antibacterial activity, concentrating our attention on investigating the quinoline moiety position, utilizing copper-catalyzed triazole formation, in order to introduce various quinoline and naphthalene derivatives. As mentioned above, copper-catalyzed triazole formation (tetrakis(acetonitrile) copper(I) hexafluorophosphate, tris[(1-benzyl$1 H$-1,2,3-triazol-4-yl)methyl]amine, $\mathrm{MeOH}$, room temperature) of 1a
Table 1 Antibacterial activity of 16-membered macrolides against 27 type strains

\begin{tabular}{|c|c|c|c|c|c|}
\hline Strain/compound & $T Y L$ & $\begin{array}{l}\text { smyco- } \\
\sin \end{array}$ & OMT & $T L M$ & Tildipirosin \\
\hline 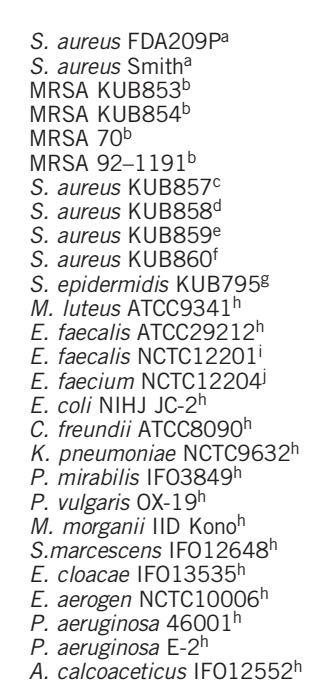 & $\begin{aligned} & 0.5 \\
& 2 \\
&> 128 \\
&> 128 \\
&> 128 \\
&> 128 \\
& 1 \\
&> 128 \\
& 64 \\
&> 128 \\
& 1 \\
& \leqslant 0.25 \\
& 1 \\
&> 128 \\
&> 128 \\
&> 128 \\
&> 128 \\
&> 128 \\
&> 128 \\
&> 128 \\
&> 128 \\
&> 128 \\
&> 128 \\
&> 128 \\
&> 128 \\
&> 128 \\
&> 128\end{aligned}$ & $\begin{aligned} & 0.5 \\
& 1 \\
&> 128 \\
&> 128 \\
&> 128 \\
&> 128 \\
& 1 \\
&> 128 \\
&> 128 \\
&> 128 \\
& 1 \\
& \leqslant 0.25 \\
& 1 \\
&> 128 \\
&> 128 \\
&> 128 \\
&> 128 \\
&> 128 \\
&> 128 \\
&> 128 \\
&> 128 \\
&> 128 \\
&> 128 \\
&> 128 \\
&> 128 \\
&> 128 \\
& 128\end{aligned}$ & $\begin{array}{c}\text { MIC } \mu g \mathrm{~m} \text { - } \\
1 \\
1 \\
>128 \\
>128 \\
>128 \\
>128 \\
2 \\
>128 \\
>128 \\
>128 \\
2 \\
\leqslant 0.25 \\
1 \\
>128 \\
>128 \\
128 \\
>128 \\
64 \\
>128 \\
>128 \\
>128 \\
>128 \\
>128 \\
>128 \\
128 \\
128 \\
>128\end{array}$ & $\begin{array}{l}0.5 \\
1 \\
>128 \\
>128 \\
>128 \\
>128 \\
0.5 \\
>128 \\
>128 \\
>128 \\
0.5 \\
\leqslant 0.25 \\
4 \\
>128 \\
>128 \\
128 \\
>128 \\
64 \\
>128 \\
>128 \\
>128 \\
>128 \\
>128 \\
>128 \\
>128 \\
>128 \\
64\end{array}$ & $\begin{array}{c}8 \\
16 \\
>128 \\
>128 \\
>128 \\
> \\
128 \\
8 \\
>128 \\
>128 \\
>128 \\
4 \\
\leqslant 0.25 \\
16 \\
>128 \\
>128 \\
8 \\
32 \\
16 \\
>128 \\
>128 \\
>128 \\
64 \\
32 \\
16 \\
>128 \\
>128 \\
32\end{array}$ \\
\hline
\end{tabular}

Abbreviation: OMT, 5-0-mycaminosyltylonolide.

as. aureus FDA209P and Smith: susceptible strains.

DMRSA KUB853, MRSA KUB854, MRSA 70, and MRSA 92-1191: MRSA strains isolated from clinical patients.

${ }^{\mathrm{C}} \mathrm{S}$. aureus KUB857: macrolide resistant strain, encoded by erm gene.

dS. aureus KUB858: macrolide resistant strain, encoded by erm gene.

e $S$. aureus KUB859: encoded by erm gene.

${ }^{\dagger} S$. aureus KUB860: encoded by erm and mef gene.

g. epidermidis KUB795: strains isolated from clinical patients.

hStandard strain.

'Enterococcus faecalis NCTC12201: encoded by van A gene.

iE. faecium NCTC12204: encoded by van $A$ gene. 


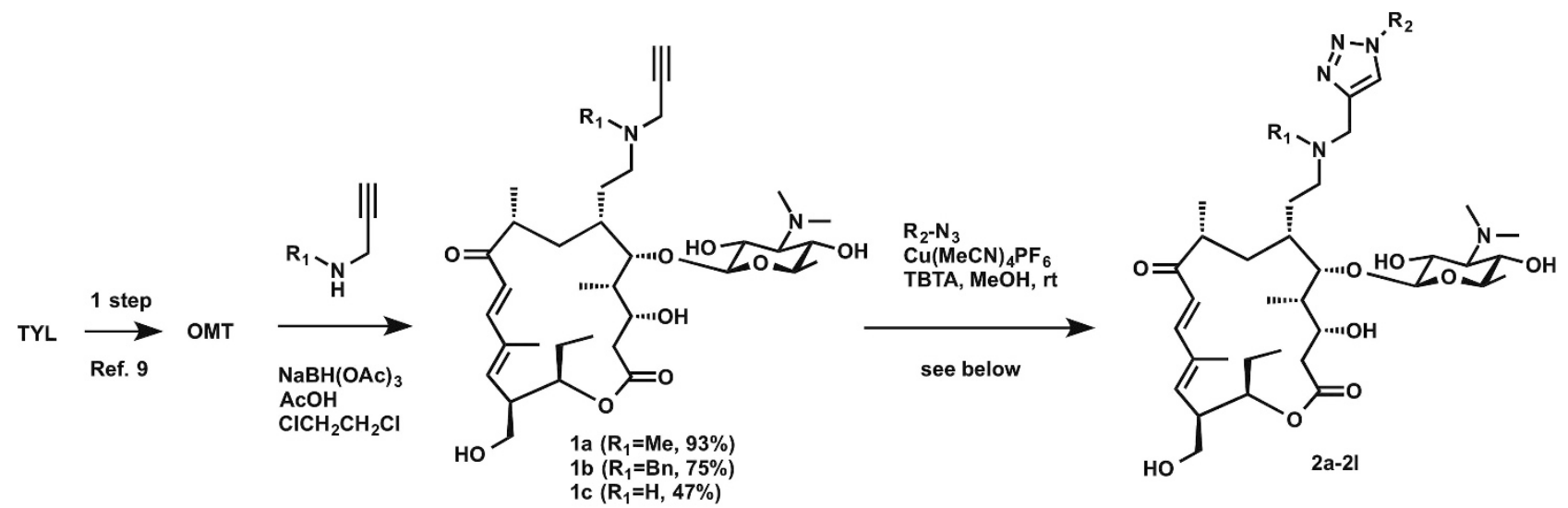<smiles>[R]CCc1ccccc1CCC(C)[R7]#N</smiles>

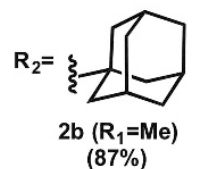<smiles>[R7]CC(C)Cc1ccccc1</smiles>

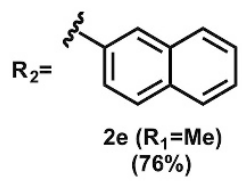

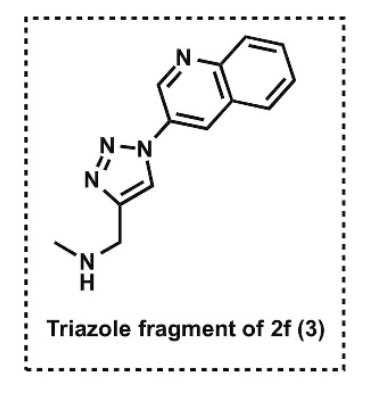

Scheme 1 Synthesis of triazole derivatives at the C20 position of OMT.

with 6 -azidoquinoline, ${ }^{18}$ 5-azidoquinoline, ${ }^{19}$ 5-azidoisoquinoline ${ }^{20}$ and 1-azidonaphthalene ${ }^{21}$ afforded the corresponding triazole products $\mathbf{2 g}$ ( $91 \%$ yield), $\mathbf{2 h}$ ( $90 \%$ yield), $\mathbf{2 i}$ (71\% yield) and $\mathbf{2 j}$ (87\% yield), respectively.

We tested analogues $\mathbf{2} \mathbf{a}-\mathbf{2} \mathbf{j}$ for in vitro activity against 27 types of bacteria, including Gram-positive and -negative strains, as well drug-susceptible and drug-resistant organisms (Table 2). ${ }^{22}$ The biological evaluation revealed that alkyne 1a (MIC $64 \mu \mathrm{g} \mathrm{ml}^{-1}$ against S. aureus) was significantly less potent against Gram-positive bacteria, compared with OMT (MIC $1 \mu \mathrm{g} \mathrm{ml}^{-1}$ against $S$. aureus). Pleasingly, a triazole derivative, 3-quinolyl $2 \mathrm{f}$ (MIC $0.25 \mu \mathrm{g} \mathrm{ml}^{-1}$ against $S$. aureus, $4 \mu \mathrm{g} \mathrm{ml}^{-1}$ against E. coli) was found to be more potent than OMT against both Gram-positive and -negative bacteria. However, the triazole fragment of $\mathbf{2} \mathbf{f}$ (3, see Experimental procedure for synthesis.) did not show any activity against all bacteria (MIC $>128 \mu \mathrm{g} \mathrm{ml}^{-1}$ ), whereas phenyl derivatives 2a, 2c, adamantyl 2b, pyridinyl 2d and nitrogen-deficient 2-naphthyl 2e displayed decreased antibacterial activity. The antibacterial activity of $\mathbf{2}$ f did not increase against resistant strains, for example, MRSA (MIC $>64 \mu \mathrm{g} \mathrm{ml}^{-1}$ against methicillin resistant staphylococcus aureus (MRSA)). The quinoline and naphthyl derivatives, $\mathbf{2} \mathbf{g}-\mathbf{2} \mathbf{j}$, showed four- to eightfold less activity than $\mathbf{2 f}$. Consequently, 3 -quinolyl $\mathbf{2} \mathbf{f}$ was found to be the most potent derivative.

Our interest then focused on investigation of $\mathrm{N}$-substituted derivatives, instead of the $\mathrm{N}$-Me group of $\mathbf{2 f}$. Reductive amination $\left(\mathrm{NaBH}(\mathrm{OAc})_{3}, \mathrm{AcOH}, \mathrm{ClCH}_{2} \mathrm{CH}_{2} \mathrm{Cl}\right)$ of OMT with $\mathrm{N}$-benzylpropargylamine and propargylamine gave $\mathbf{1 b}$ and $\mathbf{1 c}$ in $75 \%$ and $47 \%$ yields, respectively. $N$-Bn and $N-\mathrm{H}$ derivatives $2 \mathbf{k}$ and $2 \mathbf{l}$ were prepared with $\mathbf{1 b}$ and $\mathbf{1 c}$ through copper-catalyzed triazole reaction in $100 \%$ and $77 \%$ yields, respectively (Scheme 1 ). Bioactivity data indicated that $\mathbf{2 k}$ and $\mathbf{2 l}$ showed almost the same MIC values as $\mathbf{2 f}$. However,
$2 \mathbf{l}$ showed slightly less antibacterial activity, especially against $S$. aureus. Consequently, we concluded that $\mathbf{2} \mathbf{f}$ and $\mathbf{2} \mathbf{k}$ have the best balance in terms of antibacterial activity as a lead compound (Table 2).

With $\mathbf{2 k}$ as a preferred lead compound, we next investigated the effect of mycinose at the C23 position, a neutral sugar and the effect of configuration of the triazole moiety, for example, anti-triazole versus syn-triazole, with respect to antibacterial activity, respectively (Scheme 2). Reductive amination $\left(\mathrm{NaBH}(\mathrm{OAc})_{3}, \mathrm{AcOH}, \mathrm{ClCH}_{2} \mathrm{CH}_{2} \mathrm{Cl}\right)$ with desmycosin, prepared from tylosin in one step, and the quinoline triazole 4 (see Experimental procedure for synthesis), synthesized from $\mathrm{N}$-benzylpropargylamine via copper-catalyzed triazole formation $\left(\mathrm{CuSO}_{4} \cdot 7 \mathrm{H}_{2} \mathrm{O}\right.$, sodium ascorbate, $t$ - $\left.\mathrm{BuOH} / \mathrm{H}_{2} \mathrm{O}\right)$, afforded the corresponding triazole derivative $\mathbf{5}$ in $90 \%$ yield, whereas reductive amination $\left(\mathrm{NaBH}(\mathrm{OAc})_{3}, \mathrm{AcOH}, \mathrm{ClCH}_{2} \mathrm{CH}_{2} \mathrm{Cl}\right)$ with $\mathrm{OMT}$ and quinoline syn-triazole 6 (see Experimental procedure for synthesis), synthesized from $N$-benzylpropargylamine via thermal condition, afforded the corresponding syn-triazole derivative 7 in $96 \%$ yield.

Bioactivity data indicated that the mycinose-attached compound $\mathbf{5}$ possessed significantly reduced antibacterial activity against $E$. coli and K. pneumoniae, compared with $\mathbf{2 k}$ (Table 2). Likewise, syn-triazole 7 (MIC $4 \mu \mathrm{g} \mathrm{ml}^{-1}$ against $S$. aureus, $>64 \mu \mathrm{g} \mathrm{ml}^{-1}$ against E. coli) showed less activity than the anti-triazole $\mathbf{2 k}$, suggesting that anti-triazole configuration may be necessary for producing antibacterial activity. Taken together, we concluded that the hydroxyl group at the C23 position and an anti-triazole moiety are essential.

Overall, structure-activity relationship in this study were highlighted as follows (Figure 2): (1) 3-quinoline has a key role for antibacterial activity against both Gram-positive and -negative bacteria; (2) anti-triazole is better than syn; (3) $\mathrm{N}$-Me or $\mathrm{N}$-Bn substitution are more suitable than $\mathrm{N}-\mathrm{H}$ substitution; (4) mycinose removal affects increased activity. 
Table 2 Antibacterial activity of 1a-1c, 2a--2I, 3, 5 and 7 against 27 type strains

\begin{tabular}{|c|c|c|c|c|c|c|c|c|c|}
\hline Strain/compound & $1 a$ & $1 b$ & $1 c$ & $2 a$ & $2 b$ & $2 c$ & $2 d$ & $2 e$ & $2 f$ \\
\hline 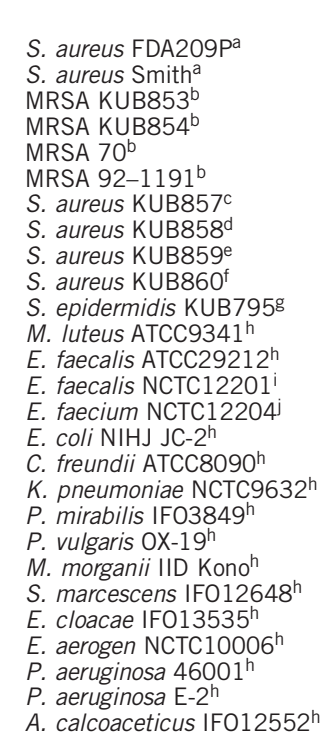 & $\begin{array}{l}64 \\
32 \\
>64 \\
>64 \\
>64 \\
>64 \\
64 \\
>64 \\
>64 \\
>64 \\
64 \\
4 \\
>64 \\
>64 \\
>64 \\
>64 \\
>64 \\
>64 \\
>64 \\
>64 \\
>64 \\
>64 \\
>64 \\
>64 \\
>64 \\
>64 \\
>64\end{array}$ & $\begin{aligned} & 8 \\
& 8 \\
> & 64 \\
> & 64 \\
> & 64 \\
> & 64 \\
& 4 \\
> & 64 \\
> & 64 \\
> & 64 \\
& 4 \\
& 1 \\
& 16 \\
> & 64 \\
> & 64 \\
> & 64 \\
> & 64 \\
> & 64 \\
> & 64 \\
> & 64 \\
> & 64 \\
> & 64 \\
> & 64 \\
> & 64 \\
> & 64 \\
> & 64 \\
> & 64\end{aligned}$ & $\begin{array}{l}32 \\
32 \\
>64 \\
>64 \\
>64 \\
>64 \\
64 \\
>64 \\
>64 \\
>64 \\
64 \\
4 \\
>64 \\
>64 \\
>64 \\
>64 \\
>64 \\
>64 \\
>64 \\
>64 \\
>64 \\
>64 \\
>64 \\
>64 \\
>64 \\
>64 \\
>64\end{array}$ & $\begin{array}{c}32 \\
16 \\
>64 \\
>64 \\
>64 \\
>64 \\
32 \\
>64 \\
>64 \\
>64 \\
32 \\
1 \\
32 \\
>64 \\
>64 \\
32 \\
>64 \\
16 \\
>64 \\
>64 \\
>64 \\
>64 \\
>64 \\
>64 \\
>64 \\
>64 \\
64\end{array}$ & $\begin{array}{c}\text { MIC } \mu g \mathrm{ml}^{-1} \\
16 \\
8 \\
>64 \\
>64 \\
>64 \\
>64 \\
64 \\
>64 \\
>64 \\
>64 \\
32 \\
2 \\
32 \\
>64 \\
>64 \\
64 \\
>64 \\
64 \\
>64 \\
>64 \\
>64 \\
>64 \\
>64 \\
>64 \\
>64 \\
>64 \\
>64\end{array}$ & $\begin{array}{c}2 \\
2 \\
>64 \\
>64 \\
>64 \\
>64 \\
4 \\
64 \\
>64 \\
>64 \\
2 \\
0.25 \\
2 \\
>64 \\
>64 \\
16 \\
32 \\
8 \\
>64 \\
64 \\
>64 \\
64 \\
>64 \\
32 \\
>64 \\
>64 \\
8\end{array}$ & $\begin{array}{c}8 \\
8 \\
>64 \\
>64 \\
>64 \\
>64 \\
16 \\
>64 \\
>64 \\
>64 \\
8 \\
0.5 \\
8 \\
>64 \\
>64 \\
32 \\
64 \\
16 \\
>64 \\
>64 \\
>64 \\
>64 \\
>64 \\
64 \\
>64 \\
>64 \\
32\end{array}$ & 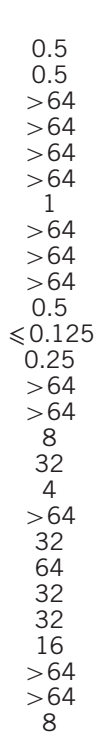 & $\begin{array}{c}0.25 \\
0.25 \\
>64 \\
>64 \\
>64 \\
>64 \\
0.25 \\
>64 \\
>64 \\
>64 \\
0.25 \\
\leqslant 0.125 \\
\leqslant 0.125 \\
>64 \\
>64 \\
4 \\
16 \\
1 \\
64 \\
8 \\
32 \\
32 \\
32 \\
16 \\
>64 \\
>64 \\
4\end{array}$ \\
\hline Strain/compound & $2 g$ & $2 h$ & $2 i$ & $2 j$ & $2 k$ & 21 & 3 & 5 & 7 \\
\hline 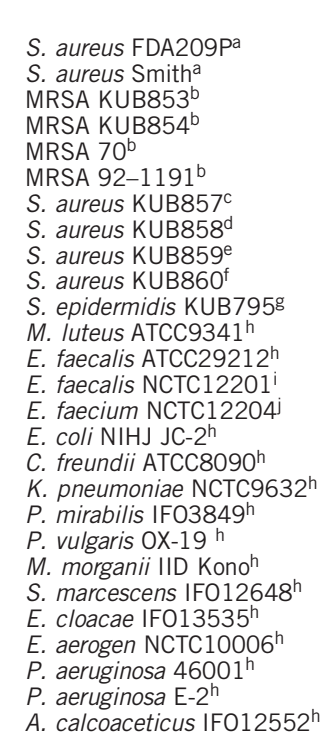 & $\begin{array}{l}1 \\
1 \\
>64 \\
>64 \\
>64 \\
>64 \\
2 \\
>64 \\
>64 \\
>64 \\
1 \\
\leqslant 0.125 \\
0.5 \\
>64 \\
>64 \\
16 \\
64 \\
8 \\
>64 \\
64 \\
>64 \\
>64 \\
64 \\
32 \\
>64 \\
>64 \\
16\end{array}$ & 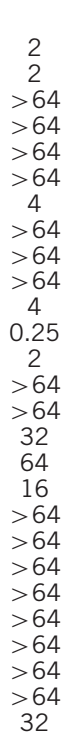 &  & 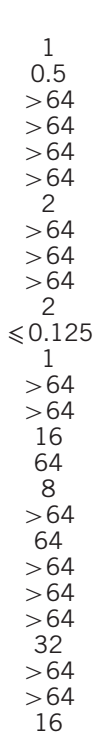 & $\begin{array}{c}\text { MIC } \mu g \mathrm{ml}^{-1} \\
\leqslant 0.125 \\
0.25 \\
>64 \\
>64 \\
>64 \\
>64 \\
0.25 \\
>64 \\
>64 \\
>64 \\
0.25 \\
\leqslant 0.125 \\
0.25 \\
>64 \\
>64 \\
16 \\
>64 \\
8 \\
64 \\
64 \\
>64 \\
>64 \\
64 \\
32 \\
>64 \\
>64 \\
16\end{array}$ & $\begin{array}{c}0.5 \\
0.5 \\
>64 \\
>64 \\
>64 \\
>64 \\
2 \\
>64 \\
>64 \\
>64 \\
2 \\
0.25 \\
0.25 \\
>64 \\
>64 \\
8 \\
32 \\
4 \\
>64 \\
32 \\
>64 \\
>64 \\
32 \\
32 \\
>64 \\
>64 \\
32\end{array}$ & $\begin{array}{l}>128 \\
>128 \\
>128 \\
>128 \\
>128 \\
>128 \\
>128 \\
>128 \\
>128 \\
>128 \\
>128 \\
>128 \\
>128 \\
>128 \\
>128 \\
>128 \\
>128 \\
>128 \\
>128 \\
>128 \\
>128 \\
>128 \\
>128 \\
>128 \\
>128 \\
>128 \\
>128\end{array}$ & $\begin{array}{c}0.5 \\
1 \\
>128 \\
>128 \\
>128 \\
>128 \\
1 \\
>128 \\
>128 \\
>128 \\
0.5 \\
\leqslant 0.25 \\
1 \\
>128 \\
>128 \\
>128 \\
>128 \\
128 \\
>128 \\
>128 \\
64 \\
>128 \\
>128 \\
>128 \\
>128 \\
>128 \\
128\end{array}$ & 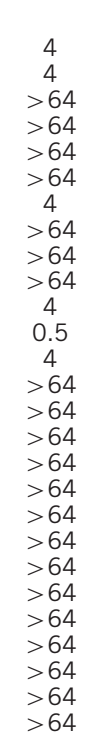 \\
\hline
\end{tabular}

${ }^{a} S$. aureus FDA209P and Smith: susceptible strains.

MRSA KUB853, MRSA KUB854, MRSA 70 and MRSA 92-1191: MRSA strains isolated from clinical patients.

'S. aureus KUB857: macrolide resistant strain, encoded by erm gene.

${ }^{\mathrm{d} S}$, aureus KUB858: macrolide resistant strain, encoded by erm gene.

es. aureus KUB859: encoded by erm gene.

S. aureus KUB860: encoded by erm and mef gene.

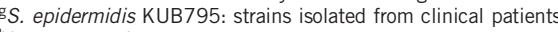

hStandard strain.

E. faecalis NCTC12201: encoded by van $A$ gene.

iE. faecium NCTC12204: encoded by van $A$ gene.

Finally, to create tylosin-based antibiotics for mastitis, we examined the antibacterial spectrum of $\mathbf{2} \mathbf{f}$ and $\mathbf{2} \mathbf{k}$ against pathogens from bovine mastitis (Table 3). In general, $\mathbf{2} \mathbf{f}$ and $\mathbf{2} \mathbf{k}$ were more potent than TLM. With respect to Staphylococci ( $S$. aureus and coagulase-negative staphylococci), 2f showed slightly better activity than TLM, whereas $2 \mathbf{k}$ showed significantly stronger activity than TLM. Especially, the $\operatorname{MIC}_{90}\left(<0.03 \mu \mathrm{g} \mathrm{ml}^{-1}\right)$ of $\mathbf{2 k}$ was $c a .30$-fold greater than the $\mathrm{MIC}_{90}$ $\left(1 \mu \mathrm{g} \mathrm{ml}^{-1}\right)$ of TLM. In terms of streptococci (S. uberis, Streptococcus dysgalactiae and Streptococcus agalactiae), $\mathbf{2} \mathbf{f}$ and $\mathbf{2 k}$ exhibited almost the same activity, or better, compared with TLM. In terms of Arcanobacterium pyogenes, the $\mathrm{MIC}_{50}\left(0.004 \mu \mathrm{g} \mathrm{ml}^{-1}\right)$ of $\mathbf{2 f}$ showed a ca. 30-fold increase compared with the $\mathrm{MIC}_{50}\left(0.125 \mu \mathrm{g} \mathrm{ml}^{-1}\right)$ of TLM. Of note, the activity against $E$. coli and $K$. pneumoniae of $2 \mathrm{f}$ was elevated 8- to 16 -fold above that of TLM $\left(\mathrm{MIC}_{50} 64-128 \mu \mathrm{g} \mathrm{ml}^{-1}\right.$ against E. coli and K. pneumoniae), whereas $2 \mathbf{k}$ exhibited 4 - to 8 -fold stronger antibacterial activity against $E$. coli than TLM $\left(\mathrm{MIC}_{50}\right.$ 

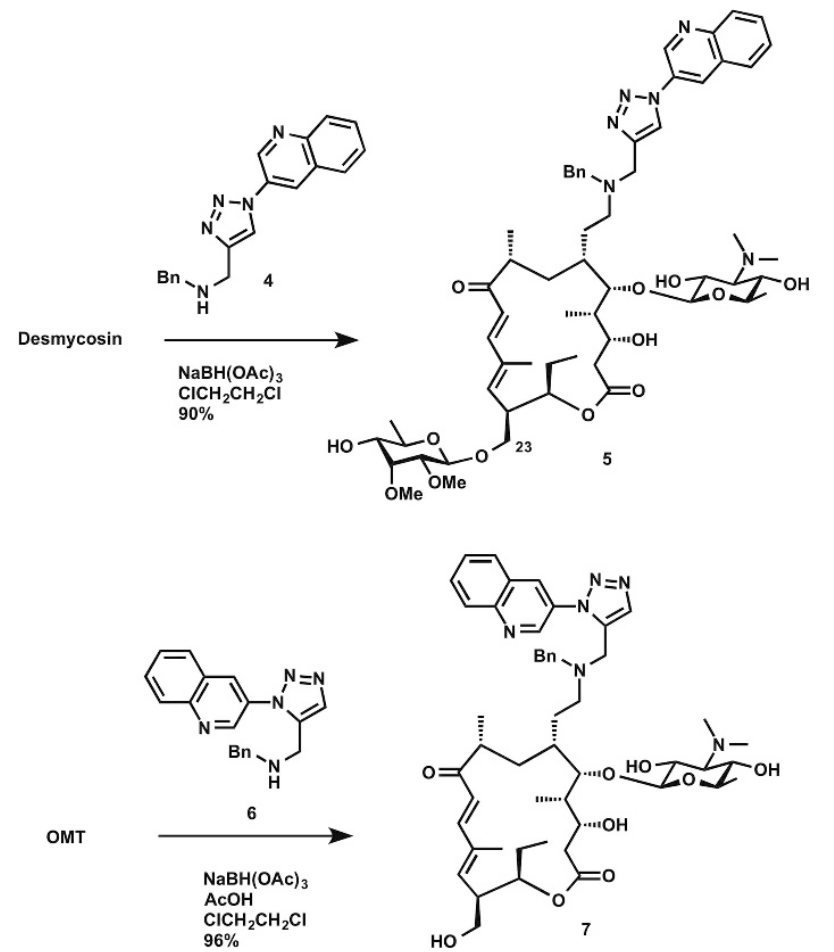

Scheme 2 Synthesis of $\mathbf{5}$ and $\mathbf{7}$.

$64 \mu \mathrm{g} \mathrm{ml}^{-1}$ ). Both the $\mathrm{MIC}_{50}$ and $\mathrm{MIC}_{90}$ of $2 \mathrm{f}$ tends to be higher than those for $\mathbf{2 k}$ against Gram-negative bacteria.

\section{CONCLUSION}

In conclusion, we developed several novel tylosin derivatives with a view to identifying a good lead compound for the development of a novel treatment for veterinary mastitis and clarified structure-activity relationships of the compounds. The antibacterial spectrum of $2 \mathbf{f}$ and $2 \mathbf{k}$ expands with regard to Gram-negative bacteria, such as E. coli and K. pneumoniae, compared with the parent compound tylosin. In addition, antibacterial activity against Gram-positive bacteria is retained or enhanced. Although the purpose of this study was to develop veterinary medicines, we believe that the results provide a useful insight for drug design, with respect to 16-membered macrolide antibiotics, for both human and animal health.

\section{EXPERIMENTAL PROCEDURES}

\section{General methods}

Analytical and preparative thin layer chromatography separations were performed using pre-coated silica gel plates with a fluorescent indicator (Merck 60 F254, Merck KGaA, Darmstadt, Germany). Flash column chromatography was performed using Kanto Chemical (60N, spherical neutral, 0.040-0.050 mm, catalog number 37563-84, Tokyo, Japan) or Merck silica gel (60N, 230-400 mesh ASTM 0.040-0.063 mm, catalog number 109385). ${ }^{1} \mathrm{H}$ NMR and ${ }^{13} \mathrm{C}$ NMR spectra were recorded at 500 and $125 \mathrm{MHz}$, respectively, using a JEOL ECA-500 spectrometer $(500 \mathrm{MHz}$, JEOL Ltd., Tokyo, Japan). Chemical shifts are expressed in p.p.m. using internal solvent peaks for $\mathrm{CDCl}_{3}\left({ }^{1} \mathrm{H}\right.$ NMR: 7.26 p.p.m.; ${ }^{13} \mathrm{C}$ NMR: 77.16 p.p.m.) and $\mathrm{CD}_{3} \mathrm{OD}\left({ }^{1} \mathrm{H}\right.$ NMR: 3.31 p.p.m.; ${ }^{13} \mathrm{C}$ NMR: 49.0 p.p.m.) as references. $J$-values are given in hertz. Coupling patterns are expressed as s (singlet), d (doublet), dd (double doublet), ddd (double double doublet), t (triplet), dt (double triplet), q (quartet), $\mathrm{m}$ (multiplet) or br (broad). All infrared spectra were measured using a Horiba

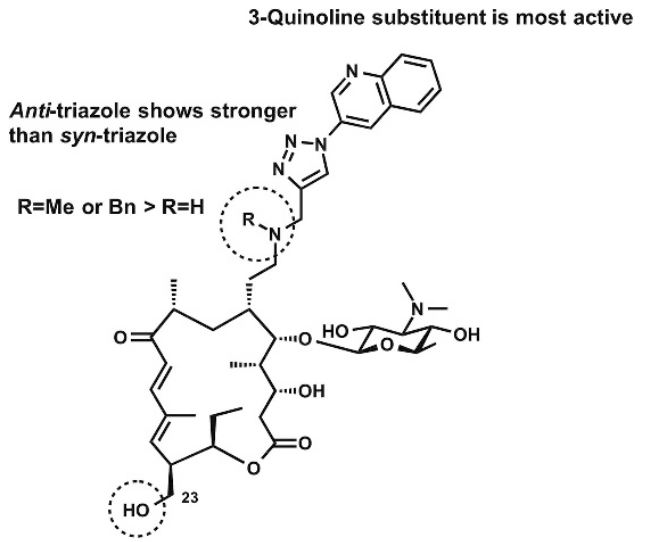

Deficiency of mycinose is necessary.

Figure 2 Structure-activity relationship (SAR) maps.

Table 3 Antibacterial activity of TLM, $2 f$ and $2 k$ for mastitis pathogens

\begin{tabular}{|c|c|c|c|c|}
\hline Strain/compound & & $T L M$ & $2 f$ & $2 k$ \\
\hline & & & $M I C \mu g m t^{-1}$ & \\
\hline \multirow[t]{3}{*}{ S.aureus $(n=12)$} & Range & $0.5-1$ & $0.125-2$ & $\leqslant 0.03$ \\
\hline & $\mathrm{MIC}_{50}$ & 0.5 & 0.25 & $\leqslant 0.03$ \\
\hline & $\mathrm{MIC}_{90}$ & 1 & 0.5 & $\leqslant 0.03$ \\
\hline \multirow[t]{3}{*}{ CNS $(n=10)$} & Range & $0.25-1$ & $\leqslant 0.03-1$ & $\leqslant 0.03$ \\
\hline & $\mathrm{MIC}_{50}$ & 0.5 & 0.25 & $\leqslant 0.03$ \\
\hline & $\mathrm{MIC}_{90}$ & 1 & 0.25 & $\leqslant 0.03$ \\
\hline \multirow[t]{3}{*}{ S.uberis $(n=12)$} & Range & $2->4$ & $0.015-0.5$ & $0.03-0.25$ \\
\hline & $\mathrm{MIC}_{50}$ & $>4$ & 0.03 & 0.06 \\
\hline & $\mathrm{MIC}_{90}$ & $>4$ & 0.06 & 0.125 \\
\hline \multirow[t]{3}{*}{ S. dysgalactiae $(n=10)$} & Range & $0.25-1$ & $0.03-0.25$ & $0.06-0.125$ \\
\hline & $\mathrm{MIC}_{50}$ & 0.25 & 0.03 & 0.06 \\
\hline & $\mathrm{MIC}_{90}$ & 0.25 & 0.06 & 0.06 \\
\hline \multirow[t]{3}{*}{ S. agalactiae $(n=10)$} & Range & $2->4$ & $0.004->4$ & $0.03->4$ \\
\hline & $\mathrm{MIC}_{50}$ & $>4$ & 0.015 & 0.06 \\
\hline & $\mathrm{MIC}_{90}$ & $>4$ & $>4$ & $>4$ \\
\hline \multirow[t]{3}{*}{ A. pyogenes $(n=10)$} & Range & $0.125-4$ & $0.004->4$ & $0.004->4$ \\
\hline & $\mathrm{MIC}_{50}$ & 0.125 & 0.004 & 0.008 \\
\hline & $\mathrm{MIC}_{90}$ & 1 & $>4$ & $>4$ \\
\hline \multirow[t]{3}{*}{ E. coli $(n=11)$} & Range & $64-128$ & $4-8$ & $8-16$ \\
\hline & $\mathrm{MIC}_{50}$ & 64 & 8 & 16 \\
\hline & $\mathrm{MIC}_{90}$ & 128 & 8 & 16 \\
\hline \multirow[t]{3}{*}{ K. pneumonia $(n=10)$} & Range & 128 & $8-16$ & $16-32$ \\
\hline & $\mathrm{MIC}_{50}$ & 128 & 16 & 32 \\
\hline & $\mathrm{MIC}_{90}$ & 128 & 16 & 32 \\
\hline
\end{tabular}

Abbreviation: CNS, coagulase-negative staphylococci.

FT-210 spectrometer (Horiba Ltd., Kyoto, Japan). High- and low-resolution mass spectra were acquired using JEOL JMS-700 MStation and JEOL JMS-T100LP instruments. Melting points were determined using a Yanaco Micro Melting Point System MP-500P (Anatec Yanaco Corporation, Kyoto, Japan).

\section{Chemicals}

All reagents were directly used as purchased, without further purification, unless otherwise noted. All new compounds were synthesized at the Kitasato Institute for Life Sciences, Kitasato University. ${ }^{1} \mathrm{H}$ and ${ }^{13} \mathrm{C}$ NMR charts of all new compounds are reported in the Supplementary Information. 


\section{Antibacterial activity measurement}

Antibacterial activities (Tables 1 and 2) of tylosin derivatives against S. aureus, Staphylococcus epidermidis, Micrococcus luteus, Enterococcus faecalis, Enterococcus faecium, E. coli, Citrobacter freundii, K. pneumoniae, Proteus mirabilis, Proteus vulgaris, Morganella morganii, Serratia marcescens, Enterobacter cloacae, Enterobacter aerogen, Pseudomonas aeruginosa and Acinetobacter calcoaceticus were investigated using the National Committee for Clinical Laboratory Standards method. (National Committee for Clinical Laboratory Standards. Performance standards for antimicrobial disk and dilution susceptibility tests for bacteria isolated from animals. Approved standard M31-A. NCCLS, Wayne, PA (1999).).

Antibacterial activities (Table 3) of tylosin derivatives against bovine mastitis isolates were determined by microbroth dilution methodology according to Clinical and Laboratory Standards Institute (Clinical and Laboratory Standards Institute. Performance standards for antimicrobial disk and dilution susceptibility tests for bacteria isolated from animals. Approved standard VET01, Clinical and Laboratory Standards Institute; Wayne, PA, USA).

\section{Experimental procedures and compound characterization}

General procedure of reductive amination. To a solution of OMT or desmycosin in 1,2-dichloroethane ( $0.1 \mathrm{M}$ for the starting materials) at room temperature was added amines (1.5 equiv.), $\mathrm{NaBH}(\mathrm{OAc})_{3}$ (1.5 equiv.) and $\mathrm{AcOH}$ (3.0 equiv.). The reaction mixture was stirred at room temperature until the starting material was consumed. To the mixture was added saturated aqueous $\mathrm{NaHCO}_{3}$. The resulting mixture was extracted with $\mathrm{CHCl}_{3}$. The combined organic layers were dried over $\mathrm{Na}_{2} \mathrm{SO}_{4}$ and concentrated in vacuo. The crude product was purified by silica gel chromatography $\left(\mathrm{CHCl}_{3} / \mathrm{MeOH} /\right.$ $30 \% \mathrm{NH}_{3}$ aq.) to give the corresponding amine derivatives.

\section{General procedure of triazole formation}

To a solution of alkyne and azide derivatives (1.2-1.5 equiv.) in $\mathrm{MeOH}(0.1 \mathrm{M}$ for alkyne) at room temperature were added tetrakis(acetonitrile) copper(I) hexafluorophosphate $(0.5 \mathrm{~mol} \%)$ and tris[(1-benzyl-1H-1,2,3-triazol-4-yl) methyl]amine $(0.5 \mathrm{~mol} \%)$. The reaction mixture was stirred at room temperature until the starting material was consumed. To the mixture was added saturated aqueous $\mathrm{NaHCO}_{3}$. The resulting mixture was extracted with $\mathrm{CHCl}_{3}$. The combined organic layers were dried over $\mathrm{Na}_{2} \mathrm{SO}_{4}$ and concentrated in vacuo. The crude product was purified by silica gel chromatography $\left(\mathrm{CHCl}_{3} / \mathrm{MeOH} / 30 \% \mathrm{NH}_{3}\right.$ aq.) to give triazole derivatives.

\section{0-Deoxy-20-( $\mathrm{N}$-methyl- $\mathrm{N}$-propargylamino)-5-O- mycaminosyltylonolide (1a)}

According to the general procedure of reductive amination, OMT $(1.00 \mathrm{~g}$, $1.67 \mathrm{mmol})$ and $N$-methylpropargylamine $(209 \mu \mathrm{l}, 2.51 \mathrm{mmol})$ were converted to $1 \mathrm{a}(1.01 \mathrm{~g}, 93 \%)$ as a pale yellow solid.

Mp: 100.6-102.0 ${ }^{\circ} \mathrm{C} ;[\alpha]^{22}{ }_{\mathrm{D}}-7.4\left(c 1.0, \mathrm{CHCl}_{3}\right) ; \mathrm{IR}(\mathrm{KBr}) \mathrm{cm}^{-1}$ : 3423, 2935, 1736, 1061, 756; ${ }^{1} \mathrm{H}$ NMR $\left(500 \mathrm{MHz}, \mathrm{CD}_{3} \mathrm{OD}\right) \delta$ (p.p.m.): 7.30 (d, $J=15.5 \mathrm{~Hz}$, $1 \mathrm{H}), 6.48(\mathrm{~d}, J=15.5 \mathrm{~Hz}, 1 \mathrm{H}), 5.93(\mathrm{~d}, J=10.3 \mathrm{~Hz}, 1 \mathrm{H}), 4.94(\mathrm{~m}, 1 \mathrm{H}), 4.28$ (d, $J=7.5 \mathrm{~Hz}, 1 \mathrm{H}), 3.77$ (d, $J=10.3 \mathrm{~Hz}, 1 \mathrm{H}), 3.69-3.67$ (complex m, 2H), 3.60 $(\mathrm{d}, J=9.7 \mathrm{~Hz}, 1 \mathrm{H}), 3.37-3.25$ (complex m, 4H), $3.13(\mathrm{~m}, 1 \mathrm{H}), 2.86(\mathrm{~m}, 1 \mathrm{H})$, $2.64(\mathrm{t}, J=2.3 \mathrm{~Hz}, 1 \mathrm{H}), 2.62-2.58$ (complex m, $2 \mathrm{H}), 2.51(\mathrm{~s}, 6 \mathrm{H}), 2.47-2.38$ (complex m, 3H), 2.27 (s, 3H), $2.05(\mathrm{~d}, J=16.6 \mathrm{~Hz}, 1 \mathrm{H}), 1.89(\mathrm{~m}, 1 \mathrm{H}), 1.86$ (s, 3H), 1.80-1.75 (complex m, 2H), 1.69-1.50 (complex m, 4H), $1.42(\mathrm{~m}, 1 \mathrm{H})$, $1.26(\mathrm{~d}, J=5.7 \mathrm{~Hz}, 3 \mathrm{H}), 1.22(\mathrm{~d}, J=6.9 \mathrm{~Hz}, 3 \mathrm{H}), 1.03(\mathrm{~d}, J=6.9 \mathrm{~Hz}, 3 \mathrm{H}), 0.95$ (t, $J=7.2 \mathrm{~Hz}, 3 \mathrm{H}) ;{ }^{13} \mathrm{C}$ NMR $\left(125 \mathrm{MHz}, \mathrm{CD}_{3} \mathrm{OD}\right) \delta$ (p.p.m.): 206.6, 174.4, $149.6,144.3,136.5,119.7,105.5,80.9,78.8,76.1,75.3,74.3,72.6,71.8,71.7$, 68.3, 62.6, 54.2, 48.2, 46.5, 45.6, 42.9, 42.2 (2C), 41.9, 40.6, 35.5, 35.0, 26.7, 26.2, 18.3, 17.9, 13.3, 10.0, 9.6; HRMS (ESI) $m / z: 651.4202[\mathrm{M}+\mathrm{H}]^{+}$, calcd. for $\mathrm{C}_{35} \mathrm{H}_{59} \mathrm{~N}_{2} \mathrm{O}_{9}: 651.4221$.

\section{0-Deoxy-20-( $N$-benzyl- $N$-propargylamino)-5-O- mycaminosyltylonolide (1b)}

According to the general procedure of reductive amination, OMT $(1.63 \mathrm{~g}$, $2.72 \mathrm{mmol})$ and crude $N$-benzylpropargylamine $(4.08 \mathrm{mmol})$ were converted to $1 \mathbf{b}(1.59 \mathrm{~g}, 75 \%)$ as a pale yellow solid.
Mp: 87.7-89.1 ${ }^{\circ} \mathrm{C} ;[\alpha]^{25} \mathrm{D}-27.0\left(\right.$ c 1.0, $\mathrm{CHCl}_{3}$ ); IR (Diamond prism) $\mathrm{cm}^{-1}$ : 3406 (br), 2927, 1716, 1589, 1057, 741; ${ }^{1} \mathrm{H}$ NMR $\left(500 \mathrm{MHz}, \mathrm{CD}_{3} \mathrm{OD}\right)$ $\delta$ (p.p.m.): 7.39-7.34 (complex m, 4H), $7.28(\mathrm{~m}, 1 \mathrm{H}), 7.24(\mathrm{~d}, J=15.5 \mathrm{~Hz}$, $1 \mathrm{H}), 6.50(\mathrm{~d}, J=15.5 \mathrm{~Hz}, 1 \mathrm{H}), 6.00(\mathrm{~d}, J=10.3 \mathrm{~Hz}, 1 \mathrm{H}), 5.04(\mathrm{~m}, 1 \mathrm{H}), 4.22$ (d, $J=7.5 \mathrm{~Hz}, 1 \mathrm{H}), 3.90(\mathrm{~d}, J=10.3 \mathrm{~Hz}, 1 \mathrm{H}), 3.77-3.65$ (complex m, $4 \mathrm{H}), 3.41$ (d, $J=12.6 \mathrm{~Hz}, 1 \mathrm{H}), 3.34(\mathrm{~m}, 1 \mathrm{H}), 3.25-3.14$ (complex m, 3H), 3.11 (app t, $J=9.5 \mathrm{~Hz}, 1 \mathrm{H}), 2.88(\mathrm{~m}, 1 \mathrm{H}), 2.76(\mathrm{~m}, 1 \mathrm{H}), 2.69-2.61$ (complex m, $2 \mathrm{H})$, 2.57-2.45 (complex m, 3H), $2.51(\mathrm{~s}, 6 \mathrm{H}), 2.38($ app t, $J=9.7 \mathrm{~Hz}, 1 \mathrm{H}), 2.09$ (d, $J=16.6 \mathrm{~Hz}, 1 \mathrm{H}), 1.96-1.48$ (complex m, $8 \mathrm{H}), 1.88$ (s, 3H), $1.22-1.92$ (complex m, 6H), $1.05(\mathrm{~d}, J=6.9 \mathrm{~Hz}, 3 \mathrm{H}), 0.97(\mathrm{t}, J=7.2 \mathrm{~Hz}, 3 \mathrm{H}) ;{ }^{13} \mathrm{C}$ NMR (125 MHz, $\left.\mathrm{CD}_{3} \mathrm{OD}\right) \delta$ (p.p.m.): 206.6, 174.4, 149.5, 144.0, 139.3, 136.6, 130.7 (2C), 129.5 (2C), 128.2, 119.9, 105.4, 80.4, 78.9, 76.0, 75.3, 74.2, 72.6, 71.71, $71.68,68.5,62.5,58.0,51.9,48.0,46.5,42.7,42.2$ (2C), 42.1, 40.7, 34.8 (2C), 26.4, 26.2, 18.2, 18.0, 13.4, 10.1, 9.6; HRMS (ESI) $\mathrm{m} / z$ : $727.4532[\mathrm{M}+\mathrm{H}]^{+}$, calcd. for $\mathrm{C}_{41} \mathrm{H}_{63} \mathrm{~N}_{2} \mathrm{O}_{9}: 727.4534$.

20-Deoxo-20- $\mathrm{N}$-propargylamino-5-O-mycaminosyltylonolide (1c) According to the general procedure of reductive amination, OMT $(100 \mathrm{mg}$, $0.167 \mathrm{mmol})$ and propargylamine $(16.1 \mu \mathrm{l}, 0.251 \mathrm{mmol})$ were converted to $1 \mathrm{c}$ (50.4 mg, $47 \%$ ) as a pale yellow solid.

Mp: $106.6-107.8^{\circ} \mathrm{C} ;[\alpha]{ }^{25} \mathrm{D}-6.0\left(\mathrm{c} 1.0, \mathrm{CHCl}_{3}\right)$; IR (Diamond prism) $\mathrm{cm}^{-1}$ : 3417(br), 2935, 1716, 1589, 1057, 752; ${ }^{1} \mathrm{H}$ NMR $\left(500 \mathrm{MHz}, \mathrm{CD}_{3} \mathrm{OD}\right)$ $\delta$ (p.p.m.): 7.31 (d, $J=15.5 \mathrm{~Hz}, 1 \mathrm{H}), 6.47$ (d, $J=15.5 \mathrm{~Hz}, 1 \mathrm{H}), 5.93$ $(\mathrm{d}, J=10.9 \mathrm{~Hz}, 1 \mathrm{H}), 4.95(\mathrm{~m}, 1 \mathrm{H}), 4.29(\mathrm{~d}, J=7.5 \mathrm{~Hz}, 1 \mathrm{H}), 3.76-3.63$ (complex m, 4H), 3.39-3.33 (complex m, 2H), $3.25(\mathrm{~m}, 1 \mathrm{H}), 3.14(\mathrm{~m}, 1 \mathrm{H})$, $2.86(\mathrm{~m}, 1 \mathrm{H}), 2.75(\mathrm{~m}, 1 \mathrm{H}), 2.67(\mathrm{~m}, 1 \mathrm{H}), 2.61(\mathrm{~m}, 1 \mathrm{H}), 2.52(\mathrm{~s}, 6 \mathrm{H})$, 2.49-2.39 (complex m, 3H), $2.05(\mathrm{~d}, J=17.2 \mathrm{~Hz}, 1 \mathrm{H}$ ), 1.95-1.38 (complex m, $8 \mathrm{H}), 1.86(\mathrm{~s}, 3 \mathrm{H}), 1.26(\mathrm{~d}, J=5.7 \mathrm{~Hz}, 3 \mathrm{H}), 1.22(\mathrm{~d}, J=6.9 \mathrm{~Hz}, 3 \mathrm{H}), 1.04$ $(\mathrm{d}, J=6.9 \mathrm{~Hz}, 3 \mathrm{H}), 0.95$ (t, $J=7.5 \mathrm{~Hz}, 3 \mathrm{H}) ;{ }^{13} \mathrm{C} \mathrm{NMR}\left(125 \mathrm{MHz}, \mathrm{CD}_{3} \mathrm{OD}\right)$ $\delta$ (p.p.m.): 206.7, 174.8, 149.8, 144.3, 136.6, 119.7, 105.4, 82.1, 80.7, 76.3, 74.3, 73.3, 72.6, 71.7 (2C), 68.1, 62.6, 48.3, 46.9, 46.5, 42.4, 42.2 (2C), 40.7, 38.0,

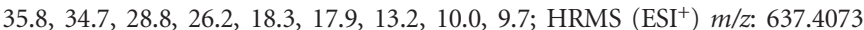
$[\mathrm{M}+\mathrm{H}]^{+}$, calcd. for $\mathrm{C}_{34} \mathrm{H}_{57} \mathrm{~N}_{2} \mathrm{O}_{9}: 637.4064$

\section{0-Deoxy-20-[ $N$-methyl- $N$-(1-benzyl-1H-1,2,3-triazol-4-yl)} methylamino]- 5-O-mycaminosyltylonolide (2a)

According to the general procedure of triazole formation, 1a $(100 \mathrm{mg}$, $0.154 \mathrm{mmol})$ and azidomethyl benzene $(30.8 \mathrm{mg}, 0.231 \mathrm{mmol})$ were converted to $\mathbf{2 a}(86.1 \mathrm{mg}, 71 \%)$ as a pale yellow solid.

Mp: $106.4-108.2^{\circ} \mathrm{C} ;[\alpha]{ }^{23} \mathrm{D}-55.9\left(\right.$ c $\left.1.0, \mathrm{CHCl}_{3}\right) ;{ }^{1} \mathrm{H}$ NMR $(500 \mathrm{MHz}$, $\left.\mathrm{CD}_{3} \mathrm{OD}\right) \delta$ (p.p.m.): 8.31 (s, 1H), 7.39-7.30 (complex m, 5H), 7.18 $(\mathrm{d}, J=15.5 \mathrm{~Hz}, 1 \mathrm{H}), 6.49(\mathrm{~d}, J=15.5 \mathrm{~Hz}, 1 \mathrm{H}), 5.85(\mathrm{~d}, J=10.3 \mathrm{~Hz}, 1 \mathrm{H})$, $5.68(\mathrm{~s}, 2 \mathrm{H}), 4.97(\mathrm{~m}, 1 \mathrm{H}), 4.20(\mathrm{~d}, J=7.5 \mathrm{~Hz}, 1 \mathrm{H}), 3.86(\mathrm{~d}, J=8.6 \mathrm{~Hz}, 1 \mathrm{H})$, $3.80(\mathrm{~d}, J=13.8,1 \mathrm{H}), 3.66-3.65$ (complex m, $2 \mathrm{H}), 3.54(\mathrm{~d}, J=10.3 \mathrm{~Hz}, 1 \mathrm{H})$, 3.36-3.32 (complex m, 2H), $3.18(\mathrm{~m}, 1 \mathrm{H}), 3.12(\mathrm{~m}, 1 \mathrm{H}), 2.86(\mathrm{~m}, 1 \mathrm{H}), 2.80$ (m, 1H), $2.64(\mathrm{~m}, 1 \mathrm{H}), 2.51(\mathrm{~s}, 6 \mathrm{H}), 2.49-2.37$ (complex m, 2H), $2.22(\mathrm{~m}, 1 \mathrm{H})$, $2.09(\mathrm{~d}, J=18.3 \mathrm{~Hz}, 1 \mathrm{H}), 2.07(\mathrm{~s}, 3 \mathrm{H}), 1.91(\mathrm{~m}, 1 \mathrm{H}), 1.85(\mathrm{~s}, 3 \mathrm{H}), 1.83-1.50$ (complex m, 7H), $1.21(\mathrm{~d}, J=6.3 \mathrm{~Hz}, 3 \mathrm{H}), 1.19(\mathrm{~d}, J=5.7 \mathrm{~Hz}, 3 \mathrm{H}), 1.02$ $(\mathrm{d}, J=6.9 \mathrm{~Hz}, 3 \mathrm{H}), 0.96(\mathrm{t}, J=7.2 \mathrm{~Hz}, 3 \mathrm{H}) ;{ }^{13} \mathrm{C} \mathrm{NMR}\left(125 \mathrm{MHz}, \mathrm{CD}_{3} \mathrm{OD}\right)$ $\delta$ (p.p.m.): 206.4, 174.4, 149.6, 145.6, 144.6, 137.2, 136.4, 130.0 (2C), 129.4, 128.8 (2C), 125.8, 119.5, 105.6, 80.4, 76.2, 74.2, 72.6, 71.73, 71.65, 68.4, 62.4, $55.7,54.8,52.5,48.2,46.6,43.0,42.4,42.2$ (2C), 40.4, 34.9, 33.9, 26.1 (2C), 18.2, 17.9, 13.3, 10.1, 9.7; HRMS $\left(\mathrm{ESI}^{+}\right) \mathrm{m} / z: 806.4673[\mathrm{M}+\mathrm{Na}]^{+}$, calcd. for $\mathrm{C}_{42} \mathrm{H}_{65} \mathrm{~N}_{5} \mathrm{NaO}_{9}$ : 806.4680.

\section{0-Deoxy-20-\{N-methyl- $N$-[1-(1-adamantyl)-1H-1,2,3-triazol-4-yl]}

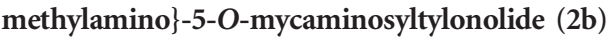

According to the general procedure of triazole formation, 1a $(100 \mathrm{mg}$, $0.154 \mathrm{mmol})$ and 1-azidoadamantane $(40.9 \mathrm{mg}, 0.230 \mathrm{mmol})$ were converted to $\mathbf{2 b}(111 \mathrm{mg}, 87 \%)$ as a pale yellow solid.

Mp: $137.0-139.0^{\circ} \mathrm{C} ;[\alpha]{ }_{\mathrm{D}}^{26}-10.9\left(c\right.$ 1.0, $\mathrm{CHCl}_{3}$ ); IR (Diamond prism) $\mathrm{cm}^{-1}:$ 2916, 1732, 1169, 1057, 748; ${ }^{1} \mathrm{H}$ NMR $\left(500 \mathrm{MHz}, \mathrm{CD}_{3} \mathrm{OD}\right) \delta$ (p.p.m.): $8.20(\mathrm{~s}, 1 \mathrm{H}), 7.29(\mathrm{~d}, J=15.5 \mathrm{~Hz}, 1 \mathrm{H}), 6.51(\mathrm{~d}, J=15.5 \mathrm{~Hz}, 1 \mathrm{H}), 5.93$ $(\mathrm{d}, J=10.9 \mathrm{~Hz}, 1 \mathrm{H}), 4.51(\mathrm{dt}, J=2.3,9.6 \mathrm{~Hz}, 1 \mathrm{H}), 4.16(\mathrm{~d}, J=7.5 \mathrm{~Hz}, 1 \mathrm{H})$, $3.83(\mathrm{~d}, J=9.7 \mathrm{~Hz}, 1 \mathrm{H}), 3.78(\mathrm{~d}, J=13.2 \mathrm{~Hz}, 1 \mathrm{H}), 3.68(\mathrm{~d}, J=5.5 \mathrm{~Hz}, 1 \mathrm{H}), 3.61$ (q, $J=6.9,7.5 \mathrm{~Hz}, 1 \mathrm{H}), 3.50(\mathrm{~d}, J=10.3 \mathrm{~Hz}, 1 \mathrm{H}), 3.44(\mathrm{~d}, J=13.2 \mathrm{~Hz}, 1 \mathrm{H})$, 
$3.34(\mathrm{~m}, 2 \mathrm{H}), 3.16(\mathrm{~m}, 1 \mathrm{H}), 3.12($ app t $, J=9.2 \mathrm{~Hz}, 1 \mathrm{H}), 2.88(\mathrm{~m}, 1 \mathrm{H}), 2.73$ (m, $1 \mathrm{H}), 2.65(\mathrm{~m}, 1 \mathrm{H}), 2.51(\mathrm{~s}, 6 \mathrm{H}), 2.44(\mathrm{dd}, J=10.0,16.9 \mathrm{~Hz}, 1 \mathrm{H}), 2.38$ $(\mathrm{t}, J=10.0 \mathrm{~Hz}, 1 \mathrm{H}), 2.33-2.16$ (complex $\mathrm{m}, 9 \mathrm{H}), 2.11$ (s, 3H), 2.08 (d, $J=16.6 \mathrm{~Hz}, 1 \mathrm{H}), 1.95-1.45$ (complex m, 18H), $1.22(\mathrm{~d}, J=6.9 \mathrm{~Hz}, 3 \mathrm{H})$, 1.19-1.16 (complex m, 6H), $1.03(\mathrm{~d}, J=6.9 \mathrm{~Hz}, 3 \mathrm{H}), 0.96(\mathrm{t}, J=7.5 \mathrm{~Hz}, 3 \mathrm{H})$; ${ }^{13} \mathrm{C}$ NMR (125 MHz, $\left.\mathrm{CD}_{3} \mathrm{OD}\right) \delta$ (p.p.m.): 206.5, 174.2, 149.6, 144.7, 143.9, $136.4,122.2,119.5,105.7,80.7,76.2,74.3,72.6,71.74,71.69,68.4,62.5,61.2$, 55.5, 53.2, 48.3, 46.6, 43.9 (3C), 43.1, 42.2 (2C), 42.1, 40.5, 37.0 (3C), 35.1, $34.3,31.0$ (3C), 26.3, 26.1, 18.3, 17.9, 13.2, 10.1, 9.7; HRMS (ESI) $\mathrm{m} / \mathrm{z}$ : $828.5474[\mathrm{M}+\mathrm{H}]^{+}$, calcd. for $\mathrm{C}_{45} \mathrm{H}_{74} \mathrm{~N}_{5} \mathrm{O}_{9}: 828.5487$.

\section{0-Deoxy-20-[ $N$-methyl- $N$-(1-phenyl-1H-1,2,3-triazol-4-yl) methylamino]-5-O-mycaminosyltylonolide (2c)}

According to the general procedure of triazole formation, 1a $(100 \mathrm{mg}$, $0.154 \mathrm{mmol})$ and phenyl azide $(22.0 \mathrm{mg}, 0.184 \mathrm{mmol})$ were converted to $2 \mathrm{c}$ (120 mg, 100\%) as a pale yellow solid.

Mp: $109.1-113.9^{\circ} \mathrm{C} ;[\alpha]{ }_{\mathrm{D}}^{26}-30.3\left(c \quad 1.0, \mathrm{CHCl}_{3}\right.$ ); IR (Diamond prism) $\mathrm{cm}^{-1}: 3352$ (br), 2931, 1732, 1589, 1061, 756; ${ }^{1} \mathrm{H}$ NMR (500 MHz, $\left.\mathrm{CD}_{3} \mathrm{OD}\right)$ $\delta$ (p.p.m.): 8.67 (s, 1H), 7.95 (m, 2H), $7.60(\mathrm{~m}, 2 \mathrm{H}), 7.50(\mathrm{~m}, 1 \mathrm{H}), 7.21$ $(\mathrm{d}, J=15.5 \mathrm{~Hz}, 1 \mathrm{H}), 6.48(\mathrm{~d}, J=15.5 \mathrm{~Hz}, 1 \mathrm{H}), 5.76(\mathrm{~d}, J=10.3 \mathrm{~Hz}, 1 \mathrm{H}), 4.84$ (m, $1 \mathrm{H}), 4.20(\mathrm{~d}, J=7.5 \mathrm{~Hz}, 1 \mathrm{H}), 3.87-3.84$ (complex m, $2 \mathrm{H}), 3.65-3.50$ (complex m, 4H), $3.34(\mathrm{dd}, J=7.5,10.3 \mathrm{~Hz}, 1 \mathrm{H}), 3.18(\mathrm{~m}, 1 \mathrm{H}), 3.11$ (app t, $J=9.5 \mathrm{~Hz}, 1 \mathrm{H}), 2.87-2.77$ (complex m, 2H), $2.66(\mathrm{~m}, 1 \mathrm{H}), 2.50(\mathrm{~s}, 6 \mathrm{H}), 2.44$ $(\mathrm{m}, 1 \mathrm{H}), 2.35(\mathrm{~m}, 1 \mathrm{H}), 2.27(\mathrm{~m}, 1 \mathrm{H}), 2.19(\mathrm{~s}, 3 \mathrm{H}), 2.06(\mathrm{~d}, J=16.6 \mathrm{~Hz}, 1 \mathrm{H})$, $1.88(\mathrm{~m}, 1 \mathrm{H}), 1.84(\mathrm{~s}, 3 \mathrm{H}), 1.82-1.50($ complex m, $7 \mathrm{H}), 1.21(\mathrm{~d}, J=6.9 \mathrm{~Hz}$, $3 \mathrm{H}), 1.20(\mathrm{~d}, J=5.7 \mathrm{~Hz}, 3 \mathrm{H}), 1.02(\mathrm{~d}, J=6.9 \mathrm{~Hz}, 3 \mathrm{H}), 0.94(\mathrm{t}, J=7.5 \mathrm{~Hz}, 3 \mathrm{H})$; ${ }^{13} \mathrm{C}$ NMR (125 MHz, $\left.\mathrm{CD}_{3} \mathrm{OD}\right) \delta$ (p.p.m.): 206.5, 174.2, 149.6, 146.0, 144.8 , $138.5,136.3,130.8$ (2C), 130.0, 123.7, 122.1 (2C), 119.4, 105.6, 80.6, 76.2, 74.3, 72.6, 71.7, 71.6, 68.3, 62.5, 55.8, 52.8, 48.3, 46.6, 43.0, 42.4, 42.2 (2C), 40.4, 35.0, 34.3, 26.2, 26.1, 18.2, 17.9, 13.2, 10.1, 9.7; HRMS (ESI) $\mathrm{m} / z: 792.4521$ $[\mathrm{M}+\mathrm{Na}]$, calcd. for $\mathrm{C}_{41} \mathrm{H}_{63} \mathrm{~N}_{5} \mathrm{O}_{9}: 792.4524$.

\section{0-Deoxy-20-\{N-methyl- $N$-[1-(3-pyridinyl)-1H-1,2,3-triazol-4-yl] methylamino\}-5-O-mycaminosyltylonolide (2d)}

According to the general procedure of triazole formation, $\mathbf{1 a}(100 \mathrm{mg}$, $0.154 \mathrm{mmol})$ and 3 -azidopyridine $(25.0 \mathrm{mg}, 0.208 \mathrm{mmol})$ were converted to 2d (118 mg, 100\%) as a pale yellow solid.

Mp: 116.0-119.2 ${ }^{\circ} \mathrm{C} ;[\alpha]{ }^{26}{ }_{\mathrm{D}}-138.6$ (c 1.0, $\mathrm{CHCl}_{3}$ ); IR (Diamond prism) $\mathrm{cm}^{-1}$ : 3402 (br), 2931, 1732, 1589, 1169, 1061, 752; ${ }^{1} \mathrm{H}$ NMR $(500 \mathrm{MHz}$, $\left.\mathrm{CD}_{3} \mathrm{OD}\right) \delta$ (p.p.m.): 9.21 (s, 1H), $8.79(\mathrm{~s}, 1 \mathrm{H}), 8.68(\mathrm{~d}, J=4.6 \mathrm{~Hz}, 1 \mathrm{H}), 8.44$ (m, 1H), $7.69(\mathrm{~m}, 1 \mathrm{H}), 7.18(\mathrm{~d}, J=15.5 \mathrm{~Hz}, 1 \mathrm{H}), 6.48(\mathrm{~d}, J=15.5 \mathrm{~Hz}, 1 \mathrm{H}), 5.74$ $(\mathrm{d}, J=10.3 \mathrm{~Hz}, 1 \mathrm{H}), 4.81(\mathrm{~m}, 1 \mathrm{H}), 4.23(\mathrm{~d}, J=7.5 \mathrm{~Hz}, 1 \mathrm{H}), 3.88-3.84$ (complex $\mathrm{m}, 2 \mathrm{H}), 3.66-3.50$ (complex m, 4H), $3.35(\mathrm{~m}, 1 \mathrm{H}), 3.22(\mathrm{~m}, 1 \mathrm{H}), 3.13(\mathrm{~m}, 1 \mathrm{H})$, 2.87-2.79 (complex m, 2H), $2.65(\mathrm{~m}, 1 \mathrm{H}), 2.50(\mathrm{~s}, 6 \mathrm{H}), 2.43(\mathrm{~m}, 1 \mathrm{H}), 2.38$ $(\mathrm{m}, 1 \mathrm{H}), 2.29(\mathrm{~m}, 1 \mathrm{H}), 2.18(\mathrm{~s}, 3 \mathrm{H}), 2.07(\mathrm{~d}, J=16.6 \mathrm{~Hz}, 1 \mathrm{H}), 1.88(\mathrm{~m}, 1 \mathrm{H})$, $1.84(\mathrm{~s}, 3 \mathrm{H}), 1.82-1.52$ (complex m, 7H), $1.22(\mathrm{~d}, J=6.9 \mathrm{~Hz}, 3 \mathrm{H}), 1.21$ $(\mathrm{d}, J=7.5 \mathrm{~Hz}, 3 \mathrm{H}), 1.02(\mathrm{~d}, J=6.9 \mathrm{~Hz}, 3 \mathrm{H}), 0.94(\mathrm{t}, J=7.2 \mathrm{~Hz}, 3 \mathrm{H}) ;{ }^{13} \mathrm{C} \mathrm{NMR}$ (125 MHz, $\mathrm{CD}_{3} \mathrm{OD}$ ) $\delta$ (p.p.m.): 206.4, 174.4, 150.5, 149.6, 146.6, 144.8, 143.0, $136.3,135.6,130.5,126.0,123.9,119.4,105.6,80.6,76.3,74.3,72.6,71.70$, $71.65,68.3,62.5,56.0,52.7,48.3,46.6,43.0,42.5,42.2$ (2C), 40.4, 34.9, 34.2, 26.2, 26.1, 18.3, 17.9, 13.2, 10.1, 9.7; HRMS (ESI) $m / z: 793.4473[\mathrm{M}+\mathrm{Na}]^{+}$, calcd. for $\mathrm{C}_{40} \mathrm{H}_{62} \mathrm{~N}_{6} \mathrm{O}_{9}$ : 793.4476 .

\section{0-Deoxy-20-\{N-methyl- $N$-[1-(2-naphthyl)-1H-1,2,3-triazol-4-yl] methylamino\}-5-O-mycaminosyltylonolide (2e)}

According to the general procedure of triazole formation, 1a $(100 \mathrm{mg}$, $0.154 \mathrm{mmol})$ and 2-azidonaphthalene $(34.5 \mathrm{mg}, 0.204 \mathrm{mmol})$ were converted to $2 \mathrm{e}(95.0 \mathrm{mg}, 76 \%)$ as a pale yellow solid.

Mp: $113.3-117.6{ }^{\circ} \mathrm{C} ;[\alpha]^{25}-16.2\left(c 1.0, \mathrm{CHCl}_{3}\right.$ ); IR (Diamond prism) $\mathrm{cm}^{-1}$ : 3450 (br), 2935, 1732, 1589, 1169, 1053, 748; ${ }^{1} \mathrm{H}$ NMR $(500 \mathrm{MHz}$, $\left.\mathrm{CD}_{3} \mathrm{OD}\right) \delta$ (p.p.m.): 8.77 (s, $\left.1 \mathrm{H}\right), 8.40(\mathrm{~s}, 1 \mathrm{H}), 8.11-8.06$ (complex m, $2 \mathrm{H}$ ), 8.02-7.97 (complex m, 2H), 7.61-7.57 (complex m, 2H), 7.16 (d, $J=15.5 \mathrm{~Hz}$, $1 \mathrm{H}), 6.46(\mathrm{~d}, J=15.5 \mathrm{~Hz}, 1 \mathrm{H}), 5.52(\mathrm{~d}, J=10.9 \mathrm{~Hz}, 1 \mathrm{H}), 4.62(\mathrm{~m}, 1 \mathrm{H}), 4.20$ (d, $J=7.5 \mathrm{~Hz}, 1 \mathrm{H}), 3.90(\mathrm{~d}, J=13.8 \mathrm{~Hz}, 1 \mathrm{H}), 3.85(\mathrm{~d}, J=9.8 \mathrm{~Hz}, 1 \mathrm{H}), 3.57$ (d, $J=10.3 \mathrm{~Hz}, 1 \mathrm{H}), 3.51(\mathrm{~d}, J=13.8 \mathrm{~Hz}, 1 \mathrm{H}), 3.38(\mathrm{dd}, J=3.4,10.9 \mathrm{~Hz}, 1 \mathrm{H})$, 3.34 (dd, $J=7.5,10.3 \mathrm{~Hz}, 1 \mathrm{H}$ ), 3.22-3.09 (complex m, 3H), $2.82(\mathrm{~m}, 1 \mathrm{H}), 2.75$ (m, $1 \mathrm{H}), 2.66(\mathrm{~m}, 1 \mathrm{H}), 2.48(\mathrm{~s}, 6 \mathrm{H}), 2.43(\mathrm{~m}, 1 \mathrm{H}), 2.34(\mathrm{~m}, 1 \mathrm{H}), 2.28(\mathrm{~m}, 1 \mathrm{H})$, $2.23(\mathrm{~s}, 3 \mathrm{H}), 2.04(\mathrm{~d}, J=17.2 \mathrm{~Hz}, 1 \mathrm{H}), 1.84-1.67$ (complex m, 5H), 1.79 (s, 3H), 1.57-1.47 (complex m, 3H), $1.22(\mathrm{~d}, J=6.3 \mathrm{~Hz}, 3 \mathrm{H}), 1.20$ (d, $J=6.9 \mathrm{~Hz}, 3 \mathrm{H}), 1.02(\mathrm{~d}, J=6.9 \mathrm{~Hz}, 3 \mathrm{H}), 0.87(\mathrm{t}, J=7.2 \mathrm{~Hz}, 3 \mathrm{H}) ;{ }^{13} \mathrm{C}$ NMR (125 MHz, $\left.\mathrm{CD}_{3} \mathrm{OD}\right) \delta$ (p.p.m.): 206.5, 174.2, 149.6, 146.2, 144.8, 136.4, 135.9, 134.6, 134.5, 131.0, 129.6, 129.0, 128.5, 128.2, 123.9, 120.6, 120.4, 119.4, $105.7,80.6,76.1,74.3,72.6,71.71,71.65,68.4,62.3,55.8,52.8,48.3,46.6,43.1$, 42.6, 42.2 (2C), 40.4, 35.0, 34.2, 26.2, 26.1, 18.2, 17.9, 13.2, 10.0, 9.7; HRMS (ESI) $m / z: 820.4852[\mathrm{M}+\mathrm{H}]^{+}$, calcd. for $\mathrm{C}_{45} \mathrm{H}_{66} \mathrm{~N}_{5} \mathrm{O}_{9}: 820.4861$.

\section{0-Deoxy-20-\{N-methyl- $N$-[1-(3-quinolyl)-1H-1,2,3-triazol-4-yl]

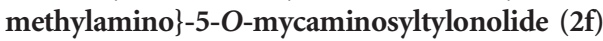

According to the general procedure of triazole formation, $1 \mathrm{a}(1.00 \mathrm{~g}$, $1.54 \mathrm{mmol}$ ) and 3 -azidoquinoline $(392 \mathrm{mg}, 2.30 \mathrm{mmol}$ ) were converted to $2 \mathrm{f}$ $(1.21 \mathrm{~g}, 96 \%)$ as a pale yellow solid.

Mp: $118.0-119.0^{\circ} \mathrm{C} ;[\alpha]{ }^{31} \mathrm{D}-114.1\left(c\right.$ 1.0, $\left.\mathrm{CHCl}_{3}\right)$; IR (Diamond prism) $\mathrm{cm}^{-1}: 3421$ (br), 2935, 1728, 1589, 1173, 1049, 756; ${ }^{1} \mathrm{H}$ NMR $(500 \mathrm{MHz}$, $\left.\mathrm{CD}_{3} \mathrm{OD}\right) \delta$ (p.p.m.): 9.49 (d, $\left.J=2.3 \mathrm{~Hz}, 1 \mathrm{H}\right), 8.91(\mathrm{~d}, J=2.3 \mathrm{~Hz}, 1 \mathrm{H}), 8.89$ (s, $1 \mathrm{H}), 8.18(\mathrm{~d}, J=8.6 \mathrm{~Hz}, 1 \mathrm{H}), 8.14(\mathrm{~d}, J=8.0 \mathrm{~Hz}, 1 \mathrm{H}), 7.90(\mathrm{~m}, 1 \mathrm{H})$, $7.75(\mathrm{~m}, 1 \mathrm{H}), 7.15(\mathrm{~d}, J=15.5 \mathrm{~Hz}, 1 \mathrm{H}), 6.48(\mathrm{~d}, J=15.5 \mathrm{~Hz}, 1 \mathrm{H}), 5.51$ $(\mathrm{d}, \quad J=10.3 \mathrm{~Hz}, 1 \mathrm{H}), \quad 4.51 \quad(\mathrm{~m}, 1 \mathrm{H}), 4.23 \quad(\mathrm{~d}, J=7.5 \mathrm{~Hz}, 1 \mathrm{H}), \quad 3.93$ (d, $J=13.8 \mathrm{~Hz}, 1 \mathrm{H}), 3.85(\mathrm{~m}, 1 \mathrm{H}), 3.59(\mathrm{~d}, J=10.3 \mathrm{~Hz}, 1 \mathrm{H}), \quad 3.52$ $(\mathrm{d}, J=13.8 \mathrm{~Hz}, 1 \mathrm{H}), 3.42(\mathrm{dd}, J=4.0,11.5 \mathrm{~Hz}, 1 \mathrm{H}), 3.35$ (dd, $J=7.5$, $10.3 \mathrm{~Hz}, 1 \mathrm{H}$ ), 3.24-3.20 (complex m, 2H), 3.13 (app t, $J=9.5 \mathrm{~Hz}, 1 \mathrm{H}$ ), 2.87 $(\mathrm{m}, 1 \mathrm{H}), 2.77(\mathrm{~m}, 1 \mathrm{H}), 2.66(\mathrm{~m}, 1 \mathrm{H}), 2.50(\mathrm{~s}, 6 \mathrm{H}), 2.45-2.29$ (complex m, 3H), $2.23(\mathrm{~s}, 3 \mathrm{H}), 2.05(\mathrm{~d}, J=16.6 \mathrm{~Hz}, 1 \mathrm{H}), 1.88-1.78$ (complex m, 3H), 1.81 (s, 3H), 1.73-1.66 (complex m, 2H), 1.58-1.45 (complex m, 3H), 1.24 (d, $J=5.7 \mathrm{~Hz}, 3 \mathrm{H}), 1.22(\mathrm{~d}, J=6.9 \mathrm{~Hz}, 3 \mathrm{H}), 1.03(\mathrm{~d}, J=6.3 \mathrm{~Hz}, 3 \mathrm{H}), 0.84$ (t, $J=7.2 \mathrm{~Hz}, 3 \mathrm{H}$ ); ${ }^{13} \mathrm{C}$ NMR (125 MHz, $\mathrm{CD}_{3} \mathrm{OD}$ ) $\delta$ (p.p.m.): 206.5, 174.3, $149.6,148.4,146.8,145.2,144.9,136.5,132.2,132.0,129.9,129.7,129.5,129.1$, $129.0,124.3,119.4,105.7,80.6,76.1,74.3,72.6,71.74,71.67,68.4,62.4,56.0$ 52.7, 48.4, 46.7, 43.1, 42.7, 42.2 (2C), 40.4, 35.0, 34.1, 26.2, 26.1, 18.3, 17.9, 13.2, 9.9, 9.7; HRMS $\left(\mathrm{ESI}^{+}\right) \mathrm{m} / z: 821.4812[\mathrm{M}+\mathrm{H}]^{+}$, calcd for $\mathrm{C}_{44} \mathrm{H}_{65} \mathrm{~N}_{6} \mathrm{O}_{9}$ : 821.4813.

\section{0-Deoxy-20-\{N-methyl- $N$-[1-(6-quinolyl)-1H-1,2,3-triazol-4-yl] methylamino\}-5-O-mycaminosyltylonolide (2g)}

According to the general procedure of triazole formation, 1a $(100 \mathrm{mg}$, $0.154 \mathrm{mmol}$ ) and 6-azidoquinoline $(39.1 \mathrm{mg}, 0.230 \mathrm{mmol})$ were converted to $2 \mathbf{g}(115 \mathrm{mg}, 91 \%)$ as a pale yellow solid.

Mp: $128.7-130.8^{\circ} \mathrm{C} ;[\alpha]^{25} \mathrm{D}-38.5$ (c 1.0, $\mathrm{CHCl}_{3}$ ); IR (Diamond prism) $\mathrm{cm}^{-1}$ : 3383 (br), 2934, 1728, 1589, 1057, 752; ${ }^{1} \mathrm{H}$ NMR (500 MHz, $\left.\mathrm{CD}_{3} \mathrm{OD}\right)$ $\delta$ (p.p.m.): 8.93 (dd, $J=1.2,4.0 \mathrm{~Hz}, 1 \mathrm{H}), 8.83$ (s, 1H), 8.52 (s, 1H), 8.51 $(\mathrm{d}, J=8.0 \mathrm{~Hz}, 1 \mathrm{H}), 8.40(\mathrm{~m}, 1 \mathrm{H}), 8.25(\mathrm{~d}, J=9.2 \mathrm{~Hz}, 1 \mathrm{H}), 7.64(\mathrm{dd}, J=4.0$, $8.0 \mathrm{~Hz}, 1 \mathrm{H}), 7.18$ (d, $J=14.9 \mathrm{~Hz}, 1 \mathrm{H}), 6.47(\mathrm{~d}, J=14.9 \mathrm{~Hz}, 1 \mathrm{H}), 5.64$ $(\mathrm{d}, J=10.3 \mathrm{~Hz}, 1 \mathrm{H}), \quad 4.69(\mathrm{~m}, 1 \mathrm{H}), 4.21 \quad(\mathrm{~d}, J=7.5 \mathrm{~Hz}, 1 \mathrm{H}), \quad 3.90$ (d, $J=13.8 \mathrm{~Hz}, 1 \mathrm{H}), 3.85(\mathrm{~d}, J=9.7 \mathrm{~Hz}, 1 \mathrm{H}), 3.58(\mathrm{~d}, J=10.3 \mathrm{~Hz}, 1 \mathrm{H}), 3.54$ $(\mathrm{d}, J=13.8 \mathrm{~Hz}, 1 \mathrm{H}), 3.47(\mathrm{dd}, J=3.4,10.9 \mathrm{~Hz}, 1 \mathrm{H}), 3.35(\mathrm{dd}, J=8.0,10.9 \mathrm{~Hz}$, $1 \mathrm{H}), 3.33(\mathrm{~m}, 1 \mathrm{H}), 3.20(\mathrm{~m}, 1 \mathrm{H}), 3.12($ app t, $J=9.5 \mathrm{~Hz}, 1 \mathrm{H}), 2.87-2.75$ (complex m, 2H), $2.66(\mathrm{~m}, 1 \mathrm{H}), 2.50(\mathrm{~s}, 6 \mathrm{H}), 2.44(\mathrm{~m}, 1 \mathrm{H}), 2.36(\mathrm{~m}, 1 \mathrm{H}), 2.29$ (m, 1H), $2.21(\mathrm{~s}, 3 \mathrm{H}), 2.05(\mathrm{~d}, J=17.2 \mathrm{~Hz}, 1 \mathrm{H}), 1.81(\mathrm{~s}, 3 \mathrm{H}), 1.84-1.76$ (complex m, 4H), $1.69(\mathrm{~m}, 1 \mathrm{H}), 1.57-1.51$ (complex m, 3H), 1.22 $(\mathrm{d}, J=5.7 \mathrm{~Hz}, 3 \mathrm{H}), 1.21(\mathrm{~d}, J=5.7 \mathrm{~Hz}, 3 \mathrm{H}), 1.03(\mathrm{~d}, J=6.9 \mathrm{~Hz}, 3 \mathrm{H}), 0.88$ (t, $J=7.5 \mathrm{~Hz}, 3 \mathrm{H}$ ); ${ }^{13} \mathrm{C}$ NMR $\left(125 \mathrm{MHz}, \mathrm{CD}_{3} \mathrm{OD}\right) \delta$ (p.p.m.): 206.5, 174.3, $152.4,149.6,148.2,146.5,144.7,138.8,136.47,136.44,131.3,130.0,124.6$, $123.9,123.8,120.3,119.5,105.7,80.6,76.1,74.3,72.5,71.7$ (2C), 68.4, 62.4, $55.8,52.9,48.2,46.6,43.1,42.5,42.2$ (2C), 40.5, 35.0, 34.2, 26.2, 26.0, 18.2, 17.9, 13.2, 10.0, 9.7; HRMS $\left(\mathrm{ESI}^{+}\right) \mathrm{m} / z: 821.4815[\mathrm{M}+\mathrm{H}]^{+}$, calcd. for $\mathrm{C}_{44} \mathrm{H}_{65} \mathrm{~N}_{6} \mathrm{O}_{9}: 821.4813$.

\section{0-Deoxy-20- $\{N$-methyl- $N$-[1-(5-quinolyl)-1H-1,2,3-triazol-4-yl] methylamino\}-5-O-mycaminosyltylonolide (2h)}

According to the general procedure of triazole formation, $\mathbf{1 a}(100 \mathrm{mg}$, $0.154 \mathrm{mmol})$ and 5-azidoquinoline $(39.1 \mathrm{mg}, 0.230 \mathrm{mmol})$ were converted to $2 \mathbf{h}(113 \mathrm{mg}, 90 \%)$ as a pale yellow solid. 
Mp: $124.1-128.8^{\circ} \mathrm{C} ;[\alpha]{ }^{27} \mathrm{D}-109.4\left(c 0.5, \mathrm{CHCl}_{3}\right)$; IR (Diamond prism) $\mathrm{cm}^{-1}$ : 3380 (br), 2935, 1732, 1589, 1169, 1057, 752; ${ }^{1} \mathrm{H}$ NMR $(500 \mathrm{MHz}$, $\left.\mathrm{CD}_{3} \mathrm{OD}\right) \delta$ (p.p.m.): $8.99(\mathrm{~m}, 1 \mathrm{H}), 8.68(\mathrm{~s}, 1 \mathrm{H}), 8.29(\mathrm{~d}, J=8.6 \mathrm{~Hz}, 1 \mathrm{H}), 8.25$ (d, $J=8.6 \mathrm{~Hz}, 1 \mathrm{H}), 8.01($ app t, $J=8.0 \mathrm{~Hz}, 1 \mathrm{H}), 7.93(\mathrm{~d}, J=7.5 \mathrm{~Hz}, 1 \mathrm{H}), 7.65$ $(\mathrm{dd}, J=4.6,8.6 \mathrm{~Hz}, 1 \mathrm{H}), 7.06(\mathrm{~d}, J=15.5 \mathrm{~Hz}, 1 \mathrm{H}), 6.42(\mathrm{~d}, J=15.5 \mathrm{~Hz}, 1 \mathrm{H})$, $5.13(\mathrm{~d}, J=10.9 \mathrm{~Hz}, 1 \mathrm{H}), 4.26(\mathrm{~d}, J=7.5 \mathrm{~Hz}, 1 \mathrm{H}), 4.05(\mathrm{~m}, 1 \mathrm{H}), 3.95$ (d, $J=13.8 \mathrm{~Hz}, 1 \mathrm{H}), 3.78(\mathrm{~d}, J=9.2 \mathrm{~Hz}, 1 \mathrm{H}), 3.57(\mathrm{~d}, J=10.3 \mathrm{~Hz}, 1 \mathrm{H}), 3.52$ (d, $J=14.3 \mathrm{~Hz}, 1 \mathrm{H}$ ), 3.49 (dd, $J=4.0,11.5 \mathrm{~Hz}, 1 \mathrm{H}), 3.36(\mathrm{dd}, J=7.5,10.3 \mathrm{~Hz}$, $1 \mathrm{H}), 3.30-3.24$ (complex m, 2H), 3.14 (app t, $J=9.5 \mathrm{~Hz}, 1 \mathrm{H}), 2.86(\mathrm{~m}, 1 \mathrm{H})$, 2.72-2.62 (complex m, 2H), 2.51 (s, 6H), 2.37-2.26 (complex m, 3H), 2.25 $(\mathrm{s}, 3 \mathrm{H}), 1.95(\mathrm{~d}, J=17.2 \mathrm{~Hz}, 1 \mathrm{H}), 1.90-1.76($ complex m, 3H), $1.74(\mathrm{~s}, 3 \mathrm{H})$, 1.68-1.63 (complex m, 2H), 1.58-1.52 (complex m, 2H), $1.44(\mathrm{~m}, 1 \mathrm{H}), 1.26$ $(\mathrm{d}, J=6.3 \mathrm{~Hz}, 3 \mathrm{H}), 1.20(\mathrm{~d}, J=6.3 \mathrm{~Hz}, 3 \mathrm{H}), 1.00(\mathrm{~d}, J=6.3 \mathrm{~Hz}, 3 \mathrm{H}), 0.76$ (t, $J=7.2 \mathrm{~Hz}, 3 \mathrm{H}) ;{ }^{13} \mathrm{C}$ NMR $\left(125 \mathrm{MHz}, \mathrm{CD}_{3} \mathrm{OD}\right) \delta$ (p.p.m.): 206.4, 173.8, 152.6, 149.6, 149.0, 145.8, 145.1, 136.2, 135.2, 133.9, 131.7, 130.4, 128.2, 125.8, 125.6, 124.1, 119.1, 105.7, 80.7, 76.1, 74.3, 72.6, 71.74, 71.69, 68.3, 62.3, 56.1, 52.4, 48.4, 46.7, 43.0, 42.8, 42.2 (2C), 40.1, 35.0, 34.0, 26.2, 25.9, 18.3, 17.9, 13.1, 10.0, 9.6; HRMS (ESI) $m / z: 821.4797[\mathrm{M}+\mathrm{H}]^{+}$, calcd. for $\mathrm{C}_{44} \mathrm{H}_{65} \mathrm{~N}_{6} \mathrm{O}_{9}$ : 821.4813 .

\section{0-Deoxy-20-\{N-methyl- $N$-[1-(5-isoquinolyl)-1H-1,2,3-triazol-4-yl $]$ methylamino\}-5-O-mycaminosyltylonolide (2i)}

According to the general procedure of triazole formation, 1a $(100 \mathrm{mg}$ $0.154 \mathrm{mmol})$ and 5-azidoisoquinoline $(39.1 \mathrm{mg}, 0.230 \mathrm{mmol})$ were converted to $2 \mathbf{i}(89.2 \mathrm{mg}, 71 \%)$ as a pale yellow solid.

Mp: $125.2-126.4{ }^{\circ} \mathrm{C} ;[\alpha]{ }^{27} \mathrm{D}-99.6\left(c\right.$ 1.0, $\mathrm{CHCl}_{3}$ ); IR (Diamond prism) $\mathrm{cm}^{-1}: 3380$ (br), 2935, 1732, 1589, 1169, 1057, 752; ${ }^{1} \mathrm{H}$ NMR $(500 \mathrm{MHz}$, $\left.\mathrm{CD}_{3} \mathrm{OD}\right) \delta$ (p.p.m.): $9.43(\mathrm{~s}, 1 \mathrm{H}), 8.70(\mathrm{~s}, 1 \mathrm{H}), 8.56(\mathrm{~d}, J=6.3 \mathrm{~Hz}, 1 \mathrm{H}), 8.38$ (d, $J=8.6 \mathrm{~Hz}, 1 \mathrm{H}), 8.11(\mathrm{~d}, J=6.9 \mathrm{~Hz}, 1 \mathrm{H}), 7.93($ app t, $J=7.7 \mathrm{~Hz}, 1 \mathrm{H}), 7.73$ (d, $J=5.7 \mathrm{~Hz}, 1 \mathrm{H}), 7.07(\mathrm{~d}, J=15.5 \mathrm{~Hz}, 1 \mathrm{H}), 6.43(\mathrm{~d}, J=14.9 \mathrm{~Hz}, 1 \mathrm{H})$, $5.22(\mathrm{~d}, J=10.3 \mathrm{~Hz}, 1 \mathrm{H}), 4.25(\mathrm{~d}, J=7.5 \mathrm{~Hz}, 1 \mathrm{H}), 4.16(\mathrm{~m}, 1 \mathrm{H}), 3.94$ $(\mathrm{dd}, J=13.2 \mathrm{~Hz}, 1 \mathrm{H}), 3.79(\mathrm{~d}, J=9.2 \mathrm{~Hz}, 1 \mathrm{H}), 3.57(\mathrm{~d}, J=10.3 \mathrm{~Hz}, 1 \mathrm{H})$, $3.55(\mathrm{~d}, J=14.3 \mathrm{~Hz}, 1 \mathrm{H}), 3.49(\mathrm{dd}, J=4.0,10.9 \mathrm{~Hz}, 1 \mathrm{H}), 3.36(\mathrm{dd}, J=7.5$, $10.3 \mathrm{~Hz}, 1 \mathrm{H}), 3.33(\mathrm{~m}, 1 \mathrm{H}), 3.26(\mathrm{~m}, 1 \mathrm{H}), 3.14(\operatorname{app~t}, J=9.5 \mathrm{~Hz}, 1 \mathrm{H}), 2.86$ (m, 1H), 2.73-2.64 (complex m, 2H), 2.51 (s, 6H), 2.41-2.28 (complex m, 3H), $2.25(\mathrm{~s}, 3 \mathrm{H}), 1.96(\mathrm{~d}, J=16.6 \mathrm{~Hz}, 1 \mathrm{H}), 1.90-1.43$ (complex m, 8H), 1.75 (s, 3H), $1.26(\mathrm{~d}, J=6.3 \mathrm{~Hz}, 3 \mathrm{H}), 1.19(\mathrm{~d}, J=6.9 \mathrm{~Hz}, 3 \mathrm{H}), 0.99(\mathrm{~d}, J=6.9 \mathrm{~Hz}$, $3 \mathrm{H}), 0.77$ (t, $J=7.2 \mathrm{~Hz}, 3 \mathrm{H}) ;{ }^{13} \mathrm{C}$ NMR $\left(125 \mathrm{MHz}, \mathrm{CD}_{3} \mathrm{OD}\right) \delta$ (p.p.m.): 206.4, 173.9, 153.7, 149.6, 145.8, 145.0, 144.8, 136.2, 134.3, 132.7, 131.4, 130.5, 129.9 , $128.6,128.1,119.2,117.6,105.7,80.7,76.1,74.3,72.6,71.72,71.67,68.3,62.3$, $56.0,52.5,48.3,46.7,43.0,42.7,42.2$ (2C), 40.1, 35.0, 34.1, 26.2, 25.9, 18.3, 17.9, 13.1, 10.0, 9.6; HRMS $\left(\mathrm{ESI}^{+}\right) \mathrm{m} / z: 821.4813[\mathrm{M}+\mathrm{H}]^{+}$, calcd. for $\mathrm{C}_{44} \mathrm{H}_{65} \mathrm{~N}_{6} \mathrm{O}_{9}: 821.4813$.

\section{0-Deoxy-20- $\{N$-methyl- $N$-[1-(1-naphthyl)-1H-1,2,3-triazol-4-yl] methylamino $-5-\mathrm{O}$-mycaminosyltylonolide $(2 \mathrm{j})$}

According to the general procedure of triazole formation, 1a $(100 \mathrm{mg}$, $0.154 \mathrm{mmol}$ ) and 1-azidonaphthalene $(39.1 \mathrm{mg}, 0.230 \mathrm{mmol})$ were converted to $2 \mathbf{j}(110 \mathrm{mg}, 87 \%)$ as a pale yellow solid.

Mp: $119.2-121.7^{\circ} \mathrm{C} ;[\alpha]{ }^{27} \mathrm{D}-93.9$ (c 1.0, $\mathrm{CHCl}_{3}$ ); IR (Diamond prism) $\mathrm{cm}^{-1}: 3398$ (br), 2931, 1732, 1173, 1053, 771; ${ }^{1} \mathrm{H}$ NMR $\left(500 \mathrm{MHz}, \mathrm{CD}_{3} \mathrm{OD}\right)$ $\delta$ (p.p.m.): 8.61 (s, 1H), 8.14 (d, $J=8.0 \mathrm{~Hz}, 1 \mathrm{H}), 8.06(\mathrm{~d}, J=7.5 \mathrm{~Hz}, 1 \mathrm{H}), 7.75$ (d, $J=7.5 \mathrm{~Hz}, 1 \mathrm{H}), 7.70(\mathrm{~m}, \quad 1 \mathrm{H}), \quad 7.65-7.60 \quad($ complex $\mathrm{m}, \quad 3 \mathrm{H}), 7.09$ $(\mathrm{d}, J=15.5 \mathrm{~Hz}, 1 \mathrm{H}), 6.42(\mathrm{~d}, J=14.9 \mathrm{~Hz}, 1 \mathrm{H}), 5.08(\mathrm{~d}, J=9.7 \mathrm{~Hz}, 1 \mathrm{H}), 4.25$ $(\mathrm{d}, J=7.5 \mathrm{~Hz}, 1 \mathrm{H}), 4.08(\mathrm{~m}, 1 \mathrm{H}), 3.96(\mathrm{~d}, J=13.8 \mathrm{~Hz}, 1 \mathrm{H}), 3.80(\mathrm{~d}, J=9.2 \mathrm{~Hz}$, $1 \mathrm{H}), 3.58(\mathrm{~d}, J=10.3 \mathrm{~Hz}, 1 \mathrm{H}), 3.53(\mathrm{~d}, J=13.8 \mathrm{~Hz}, 1 \mathrm{H}), 3.45(\mathrm{dd}, J=4.0$ $10.9 \mathrm{~Hz}, 1 \mathrm{H}$ ), 3.36 (dd, $J=7.6,10.3 \mathrm{~Hz}, 1 \mathrm{H}$ ), 3.29-3.22 (complex m, 2H), 3.14 (app t, $J=9.5 \mathrm{~Hz}, 1 \mathrm{H}), 2.86(\mathrm{~m}, 1 \mathrm{H}), 2.71-2.63($ complex m, $2 \mathrm{H}), 2.51(\mathrm{~s}, 6 \mathrm{H})$ 2.41-2.27 (complex m, 3H), 2.25 (s, 3H), $1.95(\mathrm{~d}, J=17.2 \mathrm{~Hz}, 1 \mathrm{H}), 1.90-1.77$ (complex m, 3H), $1.74(\mathrm{~s}, 3 \mathrm{H}), 1.43(\mathrm{~m}, 1 \mathrm{H}), 1.26(\mathrm{~d}, J=5.7 \mathrm{~Hz}, 3 \mathrm{H}), 1.20$ $(\mathrm{d}, J=6.3 \mathrm{~Hz}, 3 \mathrm{H}), 0.99(\mathrm{~d}, J=6.9 \mathrm{~Hz}, 3 \mathrm{H}), 0.76(\mathrm{t}, J=7.2 \mathrm{~Hz}, 3 \mathrm{H}) ;{ }^{13} \mathrm{C} \mathrm{NMR}$ $\left(125 \mathrm{MHz}, \mathrm{CD}_{3} \mathrm{OD}\right) \delta$ (p.p.m.): 206.4, 173.7, 149.7, 145.4, 145.2, 136.2, 135.6, 135.3, 131.6, 130.1, 129.4, 129.0, 128.4, 128.3, 126.3, 125.4, 123.7, 119.1, 105.7, 80.7, 76.1, 74.3, 72.6, 71.74, 71.68, 68.3, 62.4, 56.0, 52.5, 48.4, 46.7, 43.0, 42.8, 42.2 (2C), 40.1, 35.0, 34.1, 26.2, 26.0, 18.3, 17.9, 13.1, 10.0, 9.6; HRMS (ESI) $\mathrm{m} / z: 820.4840[\mathrm{M}+\mathrm{H}]^{+}$, calcd. for $\mathrm{C}_{45} \mathrm{H}_{66} \mathrm{~N}_{5} \mathrm{O}_{9}: 820.4861$.
20-Deoxy-20-\{N-benzyl- $N$-[1-(3-quinolyl)-1H-1,2,3-triazol-4-yl] methylamino\}-5-O-mycaminosyltylonolide (2k)

According to the general procedure of triazole formation, $1 \mathbf{b}(250 \mathrm{mg}$, $0.344 \mathrm{mmol}$ ) and 3 -azidoquinoline $(58.5 \mathrm{mg}, 0.413 \mathrm{mmol})$ were converted to $2 \mathbf{k}(310.5 \mathrm{mg}, 100 \%)$ as a pale yellow solid.

Mp: $118.6-120.2{ }^{\circ} \mathrm{C} ;[\alpha]^{25} \mathrm{D}-124.7\left(\right.$ c $\left.1.0, \mathrm{CHCl}_{3}\right) ;{ }^{1} \mathrm{H}$ NMR $(500 \mathrm{MHz}$, $\left.\mathrm{CD}_{3} \mathrm{OD}\right) \delta$ (p.p.m.): 9.45 (d, $\left.J=1.7 \mathrm{~Hz}, 1 \mathrm{H}\right), 8.92(\mathrm{~s}, 1 \mathrm{H}), 8.84(\mathrm{~s}, 1 \mathrm{H}), 8.12$ $(\mathrm{d}, J=8.6 \mathrm{~Hz}, 1 \mathrm{H}), 8.08(\mathrm{~d}, J=8.0 \mathrm{~Hz}, 1 \mathrm{H}), 7.83(\mathrm{~m}, 1 \mathrm{H}), 7.70(\mathrm{~m}, 1 \mathrm{H}), 7.42$ $(\mathrm{m}, 2 \mathrm{H}), 7.38(\mathrm{~m}, 2 \mathrm{H}), 7.26(\mathrm{~m}, 1 \mathrm{H}), 7.09(\mathrm{~d}, J=15.5 \mathrm{~Hz}, 1 \mathrm{H}), 6.46$ (d, $J=15.5 \mathrm{~Hz}, 1 \mathrm{H}), 5.73(\mathrm{~d}, J=10.9 \mathrm{~Hz}, 1 \mathrm{H}), 4.78(\mathrm{~m}, 1 \mathrm{H}), 4.00-3.92$ (complex m, 4H), 3.57-3.40 (complex m, 4H), $3.26(\mathrm{dd}, J=7.5,10.3 \mathrm{~Hz}, 1 \mathrm{H})$, $3.17(\mathrm{~d}, J=12.6 \mathrm{~Hz}, 1 \mathrm{H}), 3.02($ app t $J=9.5 \mathrm{~Hz}, 1 \mathrm{H}), 2.94(\mathrm{~m}, 1 \mathrm{H}), 2.84-2.78$ (complex m, 2H), $2.66(\mathrm{~m}, 1 \mathrm{H}), 2.53$ (dd, $J=10.3,17.2 \mathrm{~Hz}, 1 \mathrm{H}), 2.44(\mathrm{~s}, 6 \mathrm{H})$, 2.21-2.14 (complex m, 2H), $2.07(\mathrm{~d}, J=16.6 \mathrm{~Hz}, 1 \mathrm{H}), 1.93(\mathrm{~m}, 1 \mathrm{H}), 1.82$ (s, 3H), 1.81-1.45 (complex m, 7H), 1.19 (d, J=6.3 Hz, 3H), 1.03 $(\mathrm{d}, J=5.7 \mathrm{~Hz}, 3 \mathrm{H}), 1.03(\mathrm{~d}, J=5.7 \mathrm{~Hz}, 3 \mathrm{H}), 0.90(\mathrm{t}, J=7.5 \mathrm{~Hz}, 3 \mathrm{H}) ;{ }^{13} \mathrm{C}$ NMR (125 MHz, $\left.\mathrm{CD}_{3} \mathrm{OD}\right) \delta$ (p.p.m.): 206.6, 174.6, 149.5, 148.3, 147.7, 144.6, 144.5, 139.6, 136.6, 132.1, 131.9, 130.8 (2C), 129.8, 129.6, 129.5 (2C), 129.4, $128.9,128.3,128.2,124.2,119.6,105.6,80.4,76.3,74.2,72.5,71.6$ (2C), 68.6, $62.5,59.8,52.3,50.4,48.3,46.5,42.8,42.1$ (2C), 40.5, 34.9, 34.1, 26.2 (2C), 18.1, 17.9, 13.3, 10.0, 9.8; HRMS $\left(\mathrm{ESI}^{+}\right) \mathrm{m} / z: 897.5111[\mathrm{M}+\mathrm{H}]^{+}$, calcd. for $\mathrm{C}_{50} \mathrm{H}_{69} \mathrm{~N}_{6} \mathrm{O}_{9}: 897.5126$

\section{0-Deoxy-20-\{N-methyl- $N$-[1-(3-quinolyl)-1H-1,2,3-triazol-4-yl]} methylamino -5-O-mycaminosyltylonolide (2l)

According to the general procedure of triazole formation, 1c $(100.0 \mathrm{mg}$, $0.157 \mathrm{mmol}$ ) with 3 -azidoquinoline ( $40.1 \mathrm{mg}, 0.236 \mathrm{mmol}$ ) was converted to $21(97.9 \mathrm{mg}, 77 \%)$ as a colorless solid.

Mp: $126.5-128.0{ }^{\circ} \mathrm{C} ;[\alpha]^{26} \mathrm{D}-111.2\left(c \quad 1.0, \mathrm{CHCl}_{3}\right) ;{ }^{1} \mathrm{H}$ NMR $(500 \mathrm{MHz}$, $\left.\mathrm{CD}_{3} \mathrm{OD}\right) \delta$ (p.p.m.): 9.48 (d, $\left.J=2.9 \mathrm{~Hz}, 1 \mathrm{H}\right), 8.89$ (d, $\left.J=2.3 \mathrm{~Hz}, 1 \mathrm{H}\right), 8.82$ (s, $1 \mathrm{H}), 8.16(\mathrm{~d}, J=8.6 \mathrm{~Hz}, 1 \mathrm{H}), 8.13(\mathrm{~d}, J=8.6 \mathrm{~Hz}, 1 \mathrm{H}), 7.88(\mathrm{dt}, J=1.2$, $8.0 \mathrm{~Hz}, 1 \mathrm{H}), 7.74(\mathrm{dt}, J=1.2,8.0 \mathrm{~Hz}, 1 \mathrm{H}), 7.23(\mathrm{~d}, J=14.9 \mathrm{~Hz}, 1 \mathrm{H}), 6.48$ (d, $J=15.5 \mathrm{~Hz}, 1 \mathrm{H}), 5.70(\mathrm{~d}, J=10.3 \mathrm{~Hz}, 1 \mathrm{H}), 4.63(\operatorname{app~t}, J=8.6 \mathrm{~Hz}, 1 \mathrm{H}), 4.26$ $(\mathrm{d}, J=7.5 \mathrm{~Hz}, 1 \mathrm{H}), 4.00(\mathrm{~d}, J=14.3 \mathrm{~Hz}, 1 \mathrm{H}), 3.86(\mathrm{~d}, J=13.8 \mathrm{~Hz}, 1 \mathrm{H}), 3.80$ (d, $J=10.3 \mathrm{~Hz}, 1 \mathrm{H}), 3.65(\mathrm{~d}, J=10.3 \mathrm{~Hz}, 1 \mathrm{H}), 3.51(\mathrm{dd}, J=3.4,11.2 \mathrm{~Hz}, 1 \mathrm{H})$, 3.41-3.22 (complex m, 3H), $3.14(\mathrm{t}, J=9.2,9.7 \mathrm{~Hz}, 1 \mathrm{H}), 2.92(\mathrm{~m}, 1 \mathrm{H})$, 2.83-2.73 (complex m, 2H), $2.66(\mathrm{~m}, 1 \mathrm{H}), 2.51(\mathrm{~s}, 6 \mathrm{H}), 2.45-2.38$ (complex m, $2 \mathrm{H}), 2.04(\mathrm{~d}, J=17.2 \mathrm{~Hz}, 1 \mathrm{H}), 1.89-1.66$ (complex m, 5H), $1.82(\mathrm{~s}, 3 \mathrm{H})$, 1.60-1.45 (complex m, 3H), 1.24 (d, $J=6.3 \mathrm{~Hz}, 3 \mathrm{H}), 1.22(\mathrm{~d}, J=6.9 \mathrm{~Hz}, 3 \mathrm{H})$, $1.03(\mathrm{~d}, J=6.9 \mathrm{~Hz}, 3 \mathrm{H}), 0.84(\mathrm{t}, J=7.5 \mathrm{~Hz}, 3 \mathrm{H}) ;{ }^{13} \mathrm{C} \mathrm{NMR}\left(125 \mathrm{MHz}, \mathrm{CD}_{3} \mathrm{OD}\right)$ $\delta$ (p.p.m.): 206.6, 174.6, 149.7, 148.4, 148.3, 144.7 (2C), 136.6, 132.2, 131.9, $129.8,129.6,129.4,128.9,128.3,123.2,119.5,105.7,80.6,76.2,74.3,72.6,71.7$ (2C), 68.3, 62.5, 48.3, 47.1, 46.5, 44.5, 42.8, 42.2 (2C), 40.4, 34.7, 34.1, 27.8, 26.1, 18.2, 17.9, 13.2, 10.0, 9.7; HRMS (ESI) $m / z: 829.4478[\mathrm{M}+\mathrm{Na}]^{+}$, calcd for $\mathrm{C}_{43} \mathrm{H}_{62} \mathrm{~N}_{6} \mathrm{O}_{9} \mathrm{Na}: 829.4476$

$\mathrm{N}$-methyl- $\mathrm{N}$-[1-(3-quinolinyl)-1H-1,2,3-triazol-4-yl]methylamine (3) To a solution of 3-azidoquinoline $\left(492 \mathrm{mg}, 2.89 \mathrm{mmol}\right.$ ) in $t-\mathrm{BuOH} / \mathrm{H}_{2} \mathrm{O}$ $(29 \mathrm{ml})$ was added $N$-methylpropargylamine $(136.3 \mu \mathrm{l}, \quad 2.82 \mathrm{mmol})$, $\mathrm{CuSO}_{4} \cdot 5 \mathrm{H}_{2} \mathrm{O}(72.3 \mathrm{mg}, 0.289 \mathrm{mmol})$ and sodium L-ascorbate $(279.3 \mathrm{mg}$, $1.41 \mathrm{mmol}$ ). After being stirred for $15 \mathrm{~min}$, to the reaction mixture was added saturated aqueous Rochelle salt $(20 \mathrm{ml})$. The mixture was extracted with $\mathrm{CHCl}_{3}$ $(100 \mathrm{ml})$, dried over $\mathrm{Na}_{2} \mathrm{SO}_{4}$, filtered and concentrated in vacuo. The crude product was purified by flash column chromatography on silica gel $\left(\mathrm{CHCl}_{3} / \mathrm{MeOH}=30 / 1\right)$ to give $3(629 \mathrm{mg}, 91 \%)$ as a yellow solid.

Mp; 127.9-131.4 ${ }^{\circ} \mathrm{C}$; IR (Diamond prism) $\mathrm{cm}^{-1}$ : 3290, 3120, 3070, 810, 748; ${ }^{1} \mathrm{H}$ NMR $\left(500 \mathrm{MHz}, \mathrm{CD}_{3} \mathrm{OD}\right) \delta$ (p.p.m.): 9.39 (d, $\left.J=2.9 \mathrm{~Hz}, 1 \mathrm{H}\right), 8.77$ $(\mathrm{d}, J=2.9 \mathrm{~Hz}, 1 \mathrm{H}), 8.63(\mathrm{~s}, 1 \mathrm{H}), 8.11(\mathrm{~d}, J=8.6 \mathrm{~Hz}, 1 \mathrm{H}), 8.06(\mathrm{~d}, J=8.0 \mathrm{~Hz}$, $1 \mathrm{H}), 7.85(\mathrm{~m}, 1 \mathrm{H}), 7.71(\mathrm{~m}, 1 \mathrm{H}), 3.95(\mathrm{~s}, 2 \mathrm{H}), 2.48(\mathrm{~s}, 3 \mathrm{H}) ;{ }^{13} \mathrm{C} \mathrm{NMR}$ (125 MHz, $\left.\mathrm{CD}_{3} \mathrm{OD}\right) \delta$ (p.p.m.): 148.4, 148.0, 144.2, 132.1, 132.0, 129.7, 129.61, $129.55,129.0,127.9,122.9,46.5,35.6$; HRMS (FAB) $\mathrm{m} / z$ : found $\mathrm{m} / z: 240.1244$ $[\mathrm{M}+\mathrm{H}]^{+}$, calcd. for $\mathrm{C}_{13} \mathrm{H}_{14} \mathrm{~N}_{5}: 240.1249$. 
$N$-Benzyl- $N$-[1-(3-quinolyl)-1H-1,2,3-triazol-4-yl]methylamine (4) and $N$-Benzyl- $N$-[1-(3-quinolyl)-1H-1,2,3-triazol-5-yl]methylamine (6)
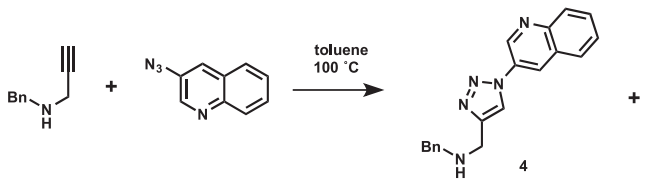

3-Azidoquinoline $(132 \mathrm{mg}, 0.773 \mathrm{mmol})$ and $\mathrm{N}$-benzylpropargylamine $(102 \mathrm{mg}, 0.702 \mathrm{mmol})$ were dissolved in toluene $(3.5 \mathrm{ml})$, and the mixture was stirred at $100{ }^{\circ} \mathrm{C}$. After stirring for $35 \mathrm{~h}$, the reaction mixture was concentrated to give the crude product. The residue was purified by flash column chromatography on silica gel $($ Hexane/EtOAc $=2 / 1$ to $1 / 2)$ to give 4 (71.6 mg, 32\%) as a brown solid and $6(43.0 \mathrm{mg}, 19 \%)$ as a brown liquid.

4; Mp; 126.6-129.0 ${ }^{\circ} \mathrm{C}$; IR (KBr) cm ${ }^{-1}: 3332,3151,2796,1496,1427,1350$, $1215,1045,741,702 ;{ }^{1} \mathrm{H}$ NMR $\left(500 \mathrm{MHz}, \mathrm{CDCl}_{3}\right) \delta$ (p.p.m.) $9.30(\mathrm{~s}, 1 \mathrm{H}), 8.47$ $(\mathrm{s}, 1 \mathrm{H}), 8.16(\mathrm{~d}, J=8.6 \mathrm{~Hz}, 1 \mathrm{H}), 8.07(\mathrm{~s}, 1 \mathrm{H}), 7.90(\mathrm{~d}, J=8.0 \mathrm{~Hz}, 1 \mathrm{H}), 7.78$ (m, 1H), $7.64(\mathrm{~m}, 1 \mathrm{H}), 7.37-7.24$ (complex m, 5H), $4.05(\mathrm{~s}, 2 \mathrm{H}), 3.90(\mathrm{~s}, 2 \mathrm{H})$, 2.05 (br s, $1 \mathrm{H}) ;{ }^{13} \mathrm{C}$ NMR $\left(125 \mathrm{MHz},\left(\mathrm{CDCl}_{3}\right) \delta\right.$ (p.p.m.) 148.3, 147.7, 143.1, $139.8,130.5,129.7,128.6,128.4$ (2C), 128.3, 128.2, 127.4, 126.1, 120.2, 52.5, 44.2; HRMS (ESI) m/z: $338.1377[\mathrm{M}+\mathrm{Na}]^{+}$, calcd. for $\mathrm{C}_{19} \mathrm{H}_{17} \mathrm{~N}_{5} \mathrm{Na}$ : 338.1382 .

6; IR (KBr) cm ${ }^{-1}$ : 3306, 2931, 1670, 1238, 1041, 748; ${ }^{1} \mathrm{H}$ NMR $(500 \mathrm{MHz}$, $\left.\mathrm{CDCl}_{3}\right) \delta$ (p.p.m.) $9.23(\mathrm{~d}, J=2.3 \mathrm{~Hz}, 1 \mathrm{H}), 8.56(\mathrm{~d}, J=2.3 \mathrm{~Hz}, 1 \mathrm{H}), 8.22$ (d, $J=8.6 \mathrm{~Hz}, 1 \mathrm{H}), 7.90(\mathrm{~d}, J=8.0 \mathrm{~Hz}, 1 \mathrm{H}), 7.85(\mathrm{~m}, 1 \mathrm{H}), 7.82(\mathrm{~s}, 1 \mathrm{H}), 7.68$ (m, 1H), 7.28-7.21 (complex m, 5H), $3.92(\mathrm{~s}, 2 \mathrm{H}), 3.82(\mathrm{~s}, 2 \mathrm{H}), 1.76(\mathrm{br} \mathrm{s}$, $1 \mathrm{H}) ;{ }^{13} \mathrm{C}$ NMR (125 MHz, $\mathrm{CDCl}_{3}$ ) $\delta$ (p.p.m.) 148.0, 146.5, 138.9, 136.7, 134.5, $131.1,131.0,130.2,129.7,128.7(2 \mathrm{C}), 128.5,128.2(2 \mathrm{C}), 128.1,127.5,53.3$, 41.4; HRMS (FAB) $m / z: 316.1557[\mathrm{M}+\mathrm{H}]^{+}$, calcd. for $\mathrm{C}_{19} \mathrm{H}_{18} \mathrm{~N}_{5}: 316.1562$.

To confirm whether compounds were 1,4- or 1,5-triazole compounds, we carried out the following reaction, as a copper-catalyzed triazole reaction exclusively gives a 1,4-triazole product.

\section{$N$-Benzyl- $N$-[1-(3-quinolyl)-1H-1,2,3-triazol-4-yl]methylamine (4)}

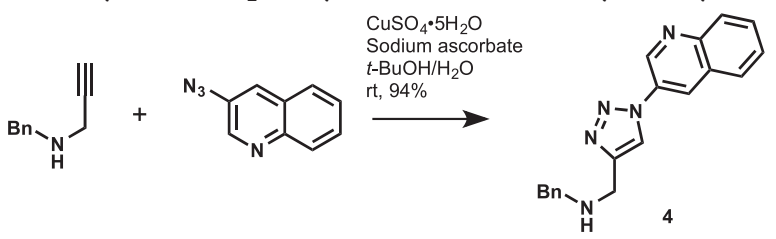

To a solution of $N$-benzylpropargylamine $(3.34 \mathrm{~g}, 23.0 \mathrm{mmol})$ and 3-azidoquinoline $(3.92 \mathrm{~g}, 23.0 \mathrm{mmol})$ in $t-\mathrm{BuOH} / \mathrm{H}_{2} \mathrm{O}=2 / 1(23 \mathrm{ml})$ were added $\mathrm{CuSO}_{4} \cdot 5 \mathrm{H}_{2} \mathrm{O} \quad(57.4 \mathrm{mg}, 0.230 \mathrm{mmol})$ and sodium L-ascorbate $(2.28 \mathrm{~g}, 11.5 \mathrm{mmol})$. The reaction mixture was stirred at room temperature. After stirring for $40 \mathrm{~min}$, to the reaction mixture was added saturated aqueous $\mathrm{NaHCO}_{3}(20 \mathrm{ml})$ and the resulting mixture was extracted with $\mathrm{CHCl}_{3}$ $(30 \mathrm{ml} \times 2)$. The combined organic layers were dried over $\mathrm{Na}_{2} \mathrm{SO}_{4}$ and concentrated at reduced pressure. The residue was recrystallized from $\mathrm{CHCl}_{3} /$ hexane at $-78{ }^{\circ} \mathrm{C}$ to give product $4(6.84 \mathrm{~g}, 94 \%)$ as a pale yellow solid.

\section{0-Deoxy-20-\{N-benzyl- $N$-[1-(3-quinolyl)-1H-1,2,3-triazol-4-yl] methylamino\}-desmycosin (5)}

According to the general procedure for reductive amination, desmycosin $(3.67 \mathrm{~g}, 4.76 \mathrm{mmol})$ and $4(1.50 \mathrm{~g}, 4.76 \mathrm{mmol})$ were converted to $5(4.60 \mathrm{~g}$, $90 \%)$ as a pale yellow solid.

Mp: $111.7-113.6^{\circ} \mathrm{C} ;[\alpha]{ }^{25} \mathrm{D}-52.3\left(c 1.0, \mathrm{CHCl}_{3}\right)$; IR (KBr) cm ${ }^{-1}: 3429$ (br), 2935, 1728, 1589, 1165, 1057, 748; ${ }^{1} \mathrm{H}$ NMR ( $\left.500 \mathrm{MHz}, \mathrm{CD}_{3} \mathrm{OD}\right) \delta$ (p.p.m.): $9.50(\mathrm{~d}, J=2.9 \mathrm{~Hz}, 1 \mathrm{H}), 8.96(\mathrm{~s}, 1 \mathrm{H}), 8.91(\mathrm{~d}, J=2.3 \mathrm{~Hz}, 1 \mathrm{H}), 8.17$ $(\mathrm{d}, J=8.6 \mathrm{~Hz}, 1 \mathrm{H}), 8.12(\mathrm{~d}, J=8.0 \mathrm{~Hz}, 1 \mathrm{H}), 7.89(\mathrm{~m}, 1 \mathrm{H}), 7.75$ (app t, $J=7.7 \mathrm{~Hz}, 1 \mathrm{H}), 7.45-7.44$ (complex m, 2H), 7.39 (app t, $J=7.5 \mathrm{~Hz}, 2 \mathrm{H}), 7.28$ (app t, $J=7.5 \mathrm{~Hz}, 1 \mathrm{H}), 7.04(\mathrm{~d}, J=15.5 \mathrm{~Hz}, 1 \mathrm{H}), 6.48(\mathrm{~d}, J=15.5 \mathrm{~Hz}, 1 \mathrm{H}), 5.71$ $(\mathrm{d}, J=10.3 \mathrm{~Hz}, 1 \mathrm{H}), 4.84(\mathrm{~m}, 1 \mathrm{H}), 4.50(\mathrm{~d}, J=8.0 \mathrm{~Hz}, 1 \mathrm{H}), 4.03-3.94$ (complex $\mathrm{m}, 4 \mathrm{H}), 3.81(\mathrm{dd}, J=4.0,9.7 \mathrm{~Hz}, 1 \mathrm{H}), 3.73(\mathrm{t}, J=2.9 \mathrm{~Hz}, 1 \mathrm{H}), 3.65(\mathrm{~m}, 1 \mathrm{H})$, $3.57(\mathrm{~s}, 3 \mathrm{H}), 3.53(\mathrm{~d}, J=10.3 \mathrm{~Hz}, 1 \mathrm{H}), 3.44(\mathrm{~d}, J=14.3 \mathrm{~Hz}, 1 \mathrm{H}), 3.40(\mathrm{~s}, 3 \mathrm{H})$, $3.34(\mathrm{~m}, 1 \mathrm{H}), 3.26(\mathrm{dd}, J=7.5,10.3 \mathrm{~Hz}, 1 \mathrm{H}), 3.19(\mathrm{~d}, J=13.2 \mathrm{~Hz}, 1 \mathrm{H}), 3.14$ (dd, $J=2.9,9.7 \mathrm{~Hz}, 1 \mathrm{H}), 3.04-2.90$ (complex m, $4 \mathrm{H}), 2.83$ (m, $1 \mathrm{H}), 2.67$ (m, 1H), 2.55 (dd, $J=10.3,17.2 \mathrm{~Hz}, 1 \mathrm{H}), 2.45$ (s, 6H), 2.22-2.13 (complex m, 2H), 2.09 (d, $J=17.2 \mathrm{~Hz}, 1 \mathrm{H}), 1.95$ (m, 1H), 1.89-1.44 (complex m, 7H), 1.82 (s, 3H), 1.22-1.20 (complex m, 6H), 1.03 (app d, $J=6.3 \mathrm{~Hz}, 6 \mathrm{H}), 0.93(\mathrm{t}$, $J=7.2 \mathrm{~Hz}, 3 \mathrm{H}) ;{ }^{13} \mathrm{C} \mathrm{NMR}\left(125 \mathrm{MHz}, \mathrm{CD}_{3} \mathrm{OD}\right) \delta$ (p.p.m.): 206.5, 174.6, 149.4, 148.3, 147.7, 144.7, 144.3, 139.6, 136.3, 132.1, 131.9, 130.8 (2C), 129.8, 129.7, 129.5 (3C), 128.9, 128.2 (2C), 124.1, 119.7, 105.6, 102.3, 82.8, 81.6, 80.3, 76.2, $74.6,74.2,72.5,71.6$ (2C), 71.0, 70.1, 68.6, 62.2, 59.8, 59.6, 52.3, 50.4, 46.5, 46.1, 42.8, 42.1 (2C), 40.5, 34.8, 34.1, 26.2, 26.1, 18.14, 18.10, 17.9, 13.3, 10.1, 9.9; HRMS (ESI) $m / z: 1071.6022[\mathrm{M}+\mathrm{H}]^{+}$, calcd. for $\mathrm{C}_{58} \mathrm{H}_{83} \mathrm{~N}_{6} \mathrm{O}_{13}: 1071.6018$.

\section{0-Deoxy-20-\{N-benzyl- $N$-[1-(3-quinolyl)-1H-1,2,3-triazol-5-yl] methylamino\}-5-O-mycaminosyltylonolide (7)}

According to the general procedure of reductive amination, OMT $(73.9 \mathrm{mg}$, $0.124 \mathrm{mmol}$ ) and $\mathbf{6}(43.0 \mathrm{mg}, 0.136 \mathrm{mmol}$ ) were converted to 7 (107 $\mathrm{mg}, 96 \%)$ as a pale yellow solid.

Mp: $108.8-111.3{ }^{\circ} \mathrm{C} ;[\alpha]{ }^{26} \mathrm{D}+4.4\left(c \quad 1.0, \mathrm{CHCl}_{3}\right.$ ); IR (Diamond prism) $\mathrm{cm}^{-1}$ : 3394 (br), 2927, 1716, 1589, 1169, 1057, 748; ${ }^{1} \mathrm{H}$ NMR $(500 \mathrm{MHz}$, $\left.\mathrm{CD}_{3} \mathrm{OD}\right) \delta$ (p.p.m.): 9.11 (d, $\left.J=2.3 \mathrm{~Hz}, 1 \mathrm{H}\right), 8.66(\mathrm{~d}, J=1.7 \mathrm{~Hz}, 1 \mathrm{H}), 8.20$ (d, $J=8.6 \mathrm{~Hz}, 1 \mathrm{H}), 8.10(\mathrm{~d}, J=8.0 \mathrm{~Hz}, 1 \mathrm{H}), 8.04(\mathrm{~s}, 1 \mathrm{H}), 7.95(\mathrm{~m}, 1 \mathrm{H}), 7.78$ (m, 1H), 7.08-7.03 (complex m, 6H), $6.42(\mathrm{~d}, J=15.5 \mathrm{~Hz}, 1 \mathrm{H}), 5.91$ (d, $J=10.3 \mathrm{~Hz}, 1 \mathrm{H}), 5.01(\mathrm{dt}, J=2.3,9.2 \mathrm{~Hz}, 1 \mathrm{H}), 4.16(\mathrm{~d}, J=7.5 \mathrm{~Hz}, 1 \mathrm{H})$, $3.89(\mathrm{~d}, J=14.9 \mathrm{~Hz}, 1 \mathrm{H}), 3.79(\mathrm{~d}, J=9.2 \mathrm{~Hz}, 1 \mathrm{H}), 3.70-3.55$ (complex m, $5 \mathrm{H})$, $3.41(\mathrm{~d}, J=13.2 \mathrm{~Hz}, 1 \mathrm{H}), 3.29(\mathrm{~m}, 1 \mathrm{H}), 3.08-3.01$ (complex m, 2H), 2.87 (m, 1H), 2.63-2.32 (complex m, 6H), $2.51(\mathrm{~s}, 6 \mathrm{H}), 2.07$ (dd, $J=1.5,17.2 \mathrm{~Hz}$, $1 \mathrm{H}), 1.91(\mathrm{~m}, 1 \mathrm{H}), 1.85(\mathrm{~s}, 3 \mathrm{H}), 1.74-1.60$ (complex m, 4H), $1.52-1.28$ (complex m, 2H), 1.14 (d, $J=6.9 \mathrm{~Hz}, 3 \mathrm{H}), 1.05-0-99$ (complex m, 6H), 0.97 (t, $J=7.5 \mathrm{~Hz}, 3 \mathrm{H}) ;{ }^{13} \mathrm{C} \mathrm{NMR}\left(125 \mathrm{MHz}, \mathrm{CD}_{3} \mathrm{OD}\right) \delta$ (p.p.m.): 206.3, 174.8, $149.3,148.7,148.1,144.1,139.2,138.6,136.6,135.5,134.1,132.5,131.4$, $130.10,130.06(2 \mathrm{C}), 129.6,129.3,129.2(2 \mathrm{C}), 128.7,128.0,119.7,105.2,80.5$, $76.4,74.2,72.5,71.7(2 \mathrm{C}), 68.6,62.7,59.7,52.8,48.2,46.8,46.5,42.5,42.2$ (2C), 40.8, 36.4, 34.5, 26.4, 26.3, 18.4, 17.8, 13.3, 10.1, 9.6; HRMS (ESI) $m / z$ : $897.5128[\mathrm{M}+\mathrm{H}]^{+}$, calcd. for $\mathrm{C}_{50} \mathrm{H}_{69} \mathrm{~N}_{6} \mathrm{O}_{9}: 897.5126$.

\section{CONFLICT OF INTEREST}

The authors declare no conflict of interest.

\section{ACKNOWLEDGEMENTS}

We thank Dr Kenichiro Nagai and Ms Noriko Sato (Kitasato University) for various instrumental analyses.

1 Ōmura, S. in: Macrolide Antibiotics. Chemistry, Biology, and Practice 2nd edn (ed. Ōmura, S.) (Academic Press, San Diego, 2002).

2 Hamill, R. L., Haney, M. E. Jr, Stamper, M. \& Wiley, P. F. Tylosin, a new antibiotic. II. Isolation, properties, and preparation of desmycosin, a microbiologically active degradation product. Antibiot. Chemother. 328-334 (1961).

3 Ōmura, S. Microbial metabolites: 45 years of wandering, wondering and discovering. Tetrahedron 67, 6420-6459 (2011).

4 Debono, M. et al. Synthesis and antimicrobial evaluation of 20-deoxo-20(3,5-dimethylpiperidin-l-yl)desmycosin (tilmicosin, EL-870) and related cyclic amino derivatives. J. Antibiot. 42, 1253-1267 (1989).

5 Miyake, T., Takita, M., Hamada, M., Takeuchi, T. \& Umezawa, S. (Zaidan Hojin Biseibutsu Kagaku Kenkyu Kai). Macrolide antibiotics and treatment of pasteurellosis. W02001016148A1 (2001).

6 Kumar, N. et al. Mastitis effects on reproductive performance in dairy cattle: a review Trop. Anim. Health Prod. 49, 663-673 (2017).

7 Gruet, P., Maincent, P., Berthelot, X. \& Kaltsatos, V. Bovine mastitis and intramammary drug delivery: review and perspectives. Adv. Drug Deliv. Rev. 50, 245-259 (2001).

8 Marshall, S. A. et al. Proposed MIC quality control guidelines for National Committee for Clinical Laboratory Standards susceptibility tests using seven veterinary antimicrobial agents: ceftiofur, enrofloxacin, florfenicol, penicillin G-novobiocin, pirlimycin, premafloxacin, and spectinomycin. J. Clin. Microbiol. 34, 2027-2029 (1996).

9 Phan, L. T. et al. 23-0-substituted 5-0-mycaminosyltylonide derivatives. W02003089446A2 (Enanta Pharmaceuticals, Inc.) (2003).

$10 \mathrm{Ma}$, Z. et al. Novel erythromycin derivatives with aryl groups tethered to the C- 6 position are potent protein synthesis inhibitors and active against multidrug-resistant respiratory pathogens. J. Med. Chem. 44, 4137-4156 (2001).

11 Tornøe, C. W., Christensen, C. \& Meldal, M. Peptidotriazoles on solid phase: [1,2,3]triazoles by regiospecific copper(I)-catalyzed 1,3-dipolar cycloadditions of terminal alkynes to azides. J. Org. Chem. 67, 3057-3064 (2002). 
12 Rostovtsev, V. V., Green, L. G., Fokin, V. V. \& Sharpless, K. B. A stepwise Huisgen cycloaddition process: copper(I)-catalyzed regioselective ligation of azides and terminal alkynes. Angew. Chem. Int. Ed. 41, 2596-2599 (2002).

13 Hirose, T. et al. Rapid 'SAR' via click chemistry: an alkyne-bearing spiramycin is fused with diverse azides to yield new triazole-antibacterial candidates. Heterocycles 69 55-61 (2006).

14 Sugawara, A. et al. Design and synthesis via click chemistry of 8,9-anhydroerythromycin A 6,9-hemiketal analogues with anti-MRSA and -VRE activity. Bioorg. Med. Chem. Lett. 17, 6340-6344 (2007).

15 Galli, U. et al. Identification of a sirtuin 3 inhibitor that displays selectivity over sirtuin 1 and 2. Eur. J. Med. Chem. 55, 58-66 (2012).

16 Lima-Neto, R. G. et al. Synthesis of 1,2,3-triazole derivatives and in vitro antifungal evaluation on Candida strains. Molecules 17, 5882-5892 (2012).

17 Sashida, H., Fujii, A. \& Tsuchiya, T. Studies on diazepines. XXIX. Syntheses of $3 \mathrm{H}$ - and $5 \mathrm{H}$-1, 4-benzodiazepines from 3-azidoquinolines. Chem. Pharm. Bull. 35, 4110-4116 (1987).
18 Ahammed, S., Saha, A. \& Ranu, B. C. Hydrogenation of azides over copper nanoparticle surface using ammonium formate in Water. J. Org. Chem. 76, 7235-7239 (2011).

19 Pokhodylo, N. T., Matiichuk, V. S. \& Obushak, N. D. Methyl 3-cyclopropyl-3oxopropanoate in the synthesis of heterocycles having a cyclopropyl substituent. Russ. J. Org. Chem. 46, 894-897 (2010).

20 Hollywood, F. et al. Photolysis of quinolyl and isoquinolyl azides in primary and secondary aliphatic amines: synthesis of bicyclic azepines, diazepines, and quinolyl- and isoquinolyl-diamines. J. Chem. Soc. Perkin Trans. 1, 421-429 (1982).

21 Mamidyala, S. K. \& Cooper, M. A. Probing the reactivity of o-phthalaldehydic acid/ methyl ester: synthesis of $\mathrm{N}$-isoindolinones and 3-arylaminophthalides. Chem. Commun. 49, 8407-8409 (2013).

22 National Committee for Clinical Laboratory Standards Performance standards for antimicrobial disk and dilution susceptibility tests for bacteria isolated from animals. Approved standard M31-A. NCCLS, Wayne, PA (1999).

Supplementary Information accompanies the paper on The Journal of Antibiotics website (http://www.nature.com/ja) 